\title{
Ruelle Zeta Function from Field Theory
}

\author{
Charles Hadfield, Santosh Kandel and Michele Schiavina®
}

\begin{abstract}
We propose a field-theoretic interpretation of Ruelle zeta function and show how it can be seen as the partition function for $B F$ theory when an unusual gauge-fixing condition on contact manifolds is imposed. This suggests an alternative rephrasing of a conjecture due to Fried on the equivalence between Ruelle zeta function and analytic torsion, in terms of homotopies of Lagrangian submanifolds.
\end{abstract}

\section{Contents}

Introduction

What to Expect from This Paper

1. Lagrangian Field Theory, the Batalin-Vilkovisky Formalism and Regularised Determinants

1.1. Classical Field Theory, Symmetries and Quantisation

1.2. Cohomological Approach and the BV Complex

1.3. Partition Functions

2. Geometric Setting

2.1. Flat Vector Bundle

2.2. Riemannian Structure and Analytic Torsion 3845

2.3. Contact Structure

2.3.1. Contact Riemannian Structure 3847

$\begin{array}{ll}\text { 2.4. Anosov Dynamics } & 3847\end{array}$

2.4.1. A Guiding Example $\quad 3848$

3. Ruelle Zeta Function 3848

3.1. Differential Forms Decomposition 3849

3.2. Ruelle Zeta Function as Regularised Determinant 3850

3.3. Meromorphic Extension of the Resolvent 3851

4. BF Theory on Contact Manifolds 3852

4.1. Analytic Torsion from Resolutions of de Rham Differential 3853

4.2. BV Interpretation: Metric Gauge for BF Theory 3856 
4.3. Contact Gauge Fixing for BF Theory

5. Lagrangian Homotopies, Fried's Conjecture and Gauge-fixing Independence

5.1. A Sphere Bundle Construction 3860

Acknowledgements

3863

References

3864

\section{Introduction}

Quantum field theory is a useful tool in many areas of pure and applied mathematics. It provides a number of precise answers, often involving insight coming from statements that are theorems in finite dimensions, and that need to be appropriately checked and generalised in infinite dimensions.

A positive example of this is the interpretation by Schwarz of the RaySinger analytic torsion in terms of a partition function for a degenerate functional $[56,57]$. The main ingredient in Schwarz's construction is a topological field theory involving differential forms, which enjoys a symmetry given by the shift of closed forms by exact ones $[8,19,28]$. This is known nowadays with the name of $B F$ theory.

From a field-theoretic point of view, such symmetry needs to be removed, or gauge fixed, as it represents a fundamental redundancy in the description. One possible way to do this is by choosing a reference metric $g$ and enforcing a $g$-dependent condition on fields. ${ }^{1}$ It allows to compute the partition function of the theory - the starting point for quantum considerations on the system - and one is left to show that the choice of metric is immaterial. The proof that such choice of metric is irrelevant was given by Schwarz for the partition function of abelian BF theory, and it is tantamount to the statement of independence of the analytic torsion on the metric used to define a Laplacian on the underlying manifold.

There are several ways of encoding a choice of gauge fixing within the framework of field theory, starting from the original idea of Faddeev and Popov [35], later understood in terms of Lie algebra cohomology by Becchi, Rouet, Stora and Tyutin [13-15,61]. A more general approach follows the ideas of by Batalin and Vilkovisky [16,17] and implements the choice of a gauge as the choice of a Lagrangian submanifold in an appropriate (graded)-symplectic manifold of fields $\mathcal{F}$. In this context, gauge-fixing independence is phrased in terms of isotopies of embedded Lagrangian submanifolds and needs to be proved in some appropriate regularisation scheme. In finite dimensions, this is a theorem: the partition function for an action functional $S$ that satisfies the quantum master equation (a differential condition on $S$ ) does not depend on the choice of a particular Lagrangian submanifold inside a smooth family $\mathbb{L}_{t} \subset \mathcal{F}$. Observe that this statement can be phrased as local constancy of the partition function w.r.t a parametrisation of the Lagrangian homotopy.

\footnotetext{
${ }^{1}$ This is often called Lorenz gauge fixing.
} 
Leaving field theory aside for a moment, consider a closed manifold $M$ endowed with an Anosov vector field (Definition 15). The flow associated with the vector field is a typical example of a dynamical system displaying "hard chaos" [43]. An important example of such a dynamical system is obtained from a Riemannian manifold $(\Sigma, g)$ whose sectional curvature is negative; then, $M=S_{g}^{*} \Sigma$, the unit cotangent bundle of $\Sigma$ (a sphere bundle), is such that the Reeb vector field $X$ associated with the natural contact structure is an Anosov vector field and its flow coincides with the geodesic flow.

If an Anosov flow (generated by the vector field $X$ ) admits closed orbits, one defines a (dynamical) Ruelle zeta function $\zeta_{X}(\lambda)$ to count lengths of closed orbits associated with the flow in a similar spirit to how the Riemann zeta function counts prime numbers $[54,55]$. The zeta function also may be defined in the presence of a representation $\rho$ of $\pi_{1}(M)$ which provides a flat vector bundle over $M$; this leads to a twisted zeta function. The chaotic nature of the dynamical system ensures that the zeta function is well defined for $\operatorname{Re}(\lambda) \gg 1$; however, some work is required to show that the function extends meromorphically to the whole complex plane [41]. Conjecture 21, due to Fried [37], proposes that when $M=S_{g}^{*} \Sigma$, the Ruelle zeta function (evaluated at zero) exactly computes the analytic torsion of the associated sphere bundle. To connect this to field theory, we observe that this means Ruelle zeta function is expected to compute - in Schwarz's terms - the partition function of $B F$ theory in a given (metric dependent) gauge fixing.

Fried's conjecture has received considerable attention recently. The proposed equality was confirmed in [37] for $\Sigma$ a hyperbolic manifold and conjectured in [38] that it also holds for compact locally symmetric spaces with nonpositive curvature. Conjecture 21, as we state it, appears in [39]. A more precise version for locally symmetric manifolds has been proved in [58] following [52]. In the variable curvature case, a perturbative result has been obtained in [32] and extended in [20]. A surprising result in the case of surfaces with variable negative curvature, but without reference to an acyclic representation, showed the zeta function at zero is determined by the topology of the surface [34]. This has been extended to the case of surfaces with boundary [45] and to higher-dimensional closed manifolds perturbatively close to hyperbolic space [47]. However, Fried's conjecture, along with its three star bounty [65, Section 3 , footnote 6], remains open.

\section{What to Expect from This Paper}

We present a new class of gauge fixings for $B F$ theory on contact manifolds based on the Reeb vector field associated with the contact structure; we call this the contact gauge in Definition 34. We then go on to show that, on sphere bundles with an Anosov-Reeb vector field, the Ruelle zeta function can be interpreted as an appropriately regularised determinant for the Lie derivative operator $\mathcal{L}_{X}$ on $k$-forms in the kernel of the contraction $\iota_{X}$. Taking this regularised determinant as the definition for the partition function of $B F$ theory in the contact gauge allows us to conclude that this coincides with the Ruelle zeta function. This point of view is analogous to Schwarz's calculation of the 
partition function of $B F$ theory (in the metric gauge), whose output is the analytic torsion, and to the recent proof of Chern-Gauss-Bonnet theorem that has been given with similar techniques in [9] (see also [23]).

As a consequence, we relate the expected gauge-fixing independence of the partition function of $B F$ theory to Fried's conjecture; in particular, we show how modern proofs of the conjecture for certain classes of manifolds (see [32]) can be taken as a proof of gauge-fixing independence. On the other hand, we believe that the field-theoretic presentation of the Ruelle zeta function provided in this paper will allow the problem to be tackled from a different angle: by means of homotopies of Lagrangian submanifolds.

To this aim, we set up a convenient construction to compare Anosov vector fields that are related to a choice of a metric on a base manifold $\Sigma$ (Sect. 5). By means of a natural construction for sphere bundles, we map smooth paths of metrics into smooth paths of Anosov vector fields, effectively constructing an isotopy between their associated Lagrangian submanifolds. This, together with the crucial local constancy results of [32], allows us to test our approach to the known case of $2 \mathrm{~d}$ surfaces - Theorem 47 provides an alternative proof of Fried's conjecture on surfaces - and interprets it as gaugefixing independence for $B F$ theory.

From the point of view of algebraic topology, this result suggests that, under certain assumptions, $\iota_{X}$ can be made into a chain contraction for the de Rham complex; namely, one can construct $\eta_{X}=\left(\mathcal{L}_{X}\right)^{-1} \iota_{X}$ with the appropriate conditions of nondegeneracy of $\mathcal{L}_{X}$. This interpretation appears to be related to the notion of a dynamical torsion introduced in [20]. There appears to be a sweet spot at the intersection of Anosov and Reeb vector fields where the independence of the "torsion" of the de Rham complex on the choice of a chain contraction and independence of the partition function of $B F$ theory on a choice of gauge fixing appear to be aspects of the same statement, expressed by Fried's conjecture.

This work is mostly addressed to the mathematical physics community working with or closely related to field theory in the Batalin-Vilkovisky formalism, but it is also aimed at the community interested in the microlocal analysis of Anosov/geodesic flows and Fried's conjecture. Therefore, we will present some basic background on field theory with symmetries to set the stage, terminology and expectations, but we will not present a complete treatment of the mathematics behind it. Results and constructions that will be somewhat assumed in this exposition of field theory can be found, for example, in $[4,24,30,31]$.

Our main goal is to present a novel link between field theory and geometric and microlocal analysis that will hopefully allow to import techniques across research fields, and stimulate fruitful interaction between scientific communities.

In Sect. 1, we give an overview on Lagrangian field theory aimed at introducing the Batalin-Vilkovisky formalism and the problem of gauge fixing. It sets the stage for the field-theoretic interpretations that will follow. 
In Sect. 2, we establishe the geometric conventions and notations, clarifying what incremental/alternative data one needs at different stages, and briefly describes the analytic torsion and Anosov dynamics.

In Sect. 3, we introduce Ruelle zeta function and its $k$-form decomposition, and state Fried's conjecture. We interpret the zeta function as a regularised (super)determinant.

In Sect. 4, we describe a field theory called $B F$ theory, and we summarise the famous interpretation (due to Schwarz) of the analytic torsion in terms of the partition function of $B F$ theory and introduce a new gauge-fixing condition on contact manifolds. We show how, with that gauge fixing, the partition function of $B F$ theory computes the Ruelle zeta function of the associated geodesic/Anosov flow.

Finally, in Sect. 5 we interpret Fried's conjecture in terms of gauge-fixing independence of $B F$ theory in the BV formalism and suggest a construction for sphere bundles that allows to present explicit homotopies between Lagrangian submanifolds.

\section{Lagrangian Field Theory, the Batalin-Vilkovisky Formalism and Regularised Determinants}

In this section, we will review the basics of the Batalin-Vilkovisky (BV) formalism [16,17] for Lagrangian field theories and how it handles gauge fixing. We will use a particular kind of regularisation based on the notion of flat traces to define determinants of operators and partition functions of quadratic functionals.

\subsection{Classical Field Theory, Symmetries and Quantisation}

The standard framework for Lagrangian field theories is as follows. To a compact manifold $M$, possibly endowed with extra geometric data, like a Riemannian metric or a contact structure, we associate a space of classical fields $F_{M}$, which is usually modelled on the space of sections of some vector bundle ${ }^{2}$ $E \rightarrow M$, together with a local functional $S_{M}$, called action functional. Local here means that it has the form of an integral over $M$ of a density-valued functional of the fields and a finite number of jets: ${ }^{3}$

$$
S_{M}=\int_{M} L_{M}\left[\phi, \partial^{I} \phi\right],
$$

where $I$ is a finite multi-index and $L_{M}$ is called Lagrangian density. For simplicity, we will consider compact manifolds without boundary, although it is possible to adapt the construction to noncompact ones or manifolds with boundary (see, for example, $[24,36]$ ).

\footnotetext{
${ }^{2}$ More generally, a sheaf.

${ }^{3}$ There is an equivalent formulation of this in the variational bi-complex [4,31], where the full jet bundle is taken into account.
} 
The dynamical content of the theory is encoded in the Euler-Lagrange locus $E L\left[S_{M}\right]$, the space of solution of the Euler-Lagrange equations coming from the variational problem for $S_{M}$. In other words, the Euler-Lagrange locus is the set of critical points of $S_{M}$.

The action functional might enjoy a symmetry. That is, it might be invariant under some transformation of the fields, for example when considering Lie algebra actions on fields taking values in Lie algebra modules. Symmetries are usually described by a (smooth) distribution $D_{M} \subset T F_{M}$, and they make the critical points of the action functional degenerate ${ }^{4}$ and will become an issue when dealing with perturbative quantisation of the theory (see below). In what follows, we will only consider symmetry distributions that are involutive.

Quantisation, loosely speaking, is meant to replace the (commutative) algebra of functions over the space of physical configurations of the system with some (noncommutative) algebra of operators over a suitable vector space, also called the space of quantum states. Without delving too much into how this is achieved in general, for our purposes it will be important to mention that one possible procedure starts by making sense of the following expression:

$$
Z=\int \exp \left(\frac{i}{\hbar} S_{M}\right),
$$

usually called the partition function, where the integral sign should ideally represent actual integration over $F_{M}$, with some measure. However, an appropriate integration theory for such (infinite-dimensional) spaces of fields is generally not available, and one defines the previous expression as a formal power series expansion in the parameter $\hbar$. This approach, however, requires the critical points of $S_{M}$ to be isolated, as it involves a saddle point or stationary phase approximation around critical points. It therefore automatically fails in the presence of symmetries, unless appropriate prescriptions are enforced.

We choose to deal with this problem by means of the Batalin-Vilkovisky (BV) formalism.

\subsection{Cohomological Approach and the BV Complex}

Degenerate functionals are usually accompanied by involutive (symmetry) distributions $D_{M}$, and the space of inequivalent field configurations is the quotient $E L\left[S_{M}\right] / D_{M}$. Most of the times the quotient is singular, and one looks for a replacement for it.

A resolution of $E L\left[S_{M}\right] / D_{M}$ is given by a complex $\left(C^{\bullet}, d_{C}\right)$ such that ${ }^{5}$ for all $i>0$

$$
H^{-i}\left(C^{\bullet}\right)=0, \quad H^{0}\left(C^{\bullet}\right) \simeq C^{\infty}\left(E L\left[S_{M}\right] / D_{M}\right) .
$$

One way to obtain a resolution is by first localising to the submanifold $E L\left[S_{M}\right]$ constructing the Koszul-Tate complex and then following the Chevalley-Eilenberg procedure to describe $D_{M}$-invariant functions on it (see

\footnotetext{
${ }^{4}$ We will only be concerned with continuous symmetries.

${ }^{5}$ In some practical cases, the vanishing of the negative cohomology is not guaranteed. We will anyway not need this condition in what follows.
} 
[59] for a "geometric" jet-bundle explanation of this and [36] for a more "algebraic" one).

The Batalin-Vilkovisky formalism is essentially the interpretation of said complex as the space of functions over a $(-1)$-symplectic graded manifold $\left(\mathcal{F}_{B V}, \Omega_{B V}\right)[24,25,29,46,59]$, whose degree-0 part coincides with the original space of fields $F_{M}$, endowed with an odd vector field of degree-1 $Q \in$ $C^{\infty}\left(\mathcal{F}_{B V}, T[1] \mathcal{F}_{B V}\right)$ such that ${ }^{6}[Q, Q]=0$ and a degree-0 functional $\mathcal{S}_{M}$ : $\mathcal{F}_{B V} \rightarrow \mathbb{R}$ satisfying

$$
\iota_{Q} \iota_{Q} \Omega_{B V}=\left\{\mathcal{S}_{M}, \mathcal{S}_{M}\right\}_{\Omega_{B V}}=0,
$$

with $\{\cdot, \cdot\}_{\Omega_{B V}}$ the Poisson bracket associated with the symplectic structure $\Omega_{B V}$, and the compatibility condition

$$
\iota_{Q} \Omega_{B V}=d \mathcal{S}_{M} .
$$

Remark 1. In infinite dimensions, one models $\mathcal{F}_{B V}$ on some appropriate space of sections of the jet bundle of a vector bundle $E \rightarrow M$. Then, the de Rham differential in Eq. (5) is replaced with $\delta$, the variation operator interpreted as the vertical differential on local functionals over $\mathcal{F}_{B V}$ (see [4]). Observe that we could take Eq. (4) as a definition of Poisson brackets in infinite dimensions.

On $C^{\infty}\left(\mathcal{F}_{B V}\right)$, one constructs another (second order) differential $\left(\Delta_{B V}^{2}=\right.$ 0 ) called BV-Laplacian and defines gauge fixing to be the choice of a Lagrangian submanifold $\mathbb{L} \subset \mathcal{F}_{B V}$. The main results are as follows:

Theorem 2. Let $\left(\mathcal{F}_{B V}, \Omega_{B V}\right)$ be a finite-dimensional (-1)-symplectic graded manifold, with a measure $\mu$ and the $B V$ Laplacian $\Delta_{\mu}$, a coboundary operator defined on $C^{\infty}\left(\mathcal{F}_{B V}\right)$ such that for all $f \in C^{\infty}\left(\mathcal{F}_{B V}\right)$ we have

$$
\Delta_{\mu} f=-\frac{1}{2} \operatorname{div}_{\mu}\left(X_{f}\right)
$$

with $X_{f}$ the Hamiltonian vector field of $f$ with respect to $\Omega_{B V}$. Assuming that $\Delta_{\mu_{B V}} f=0$ and $g=\Delta_{\mu_{B V}} h$, with $f, g, h \in C^{\infty}\left(\mathcal{F}_{B V}\right)$ then:

- for any Lagrangian submanifold $\mathbb{L} \subset \mathcal{F}_{B V}$

$$
\left.\int_{\mathbb{L}} g \mu_{B V}\right|_{\mathbb{L}}=0 ;
$$

- given a continuous family of Lagrangian submanifolds $\mathbb{L}_{t}$

$$
\left.\frac{d}{\mathrm{~d} t} \int_{\mathbb{L}_{t}} f \mu_{B V}\right|_{\mathbb{L}_{t}}=0 .
$$

Remark 3. The definition of the BV Laplacian in (6) implies the relations

$$
\begin{aligned}
\Delta_{\mu}(f g) & =\left(\Delta_{\mu} f\right) g+(-1)^{|f|} f\left(\Delta_{\mu} g\right)+(-1)^{|f|}\{f, g\}_{\Omega_{B V}} \\
\Delta_{\mu}\{f, g\}_{\Omega_{B V}} & =\left\{\Delta_{\mu} f, g\right\}_{\Omega_{B V}}+(-1)^{|f|+1}\left\{f, \Delta_{\mu} g\right\}_{\Omega_{B V}},
\end{aligned}
$$

making the tuple $\left(C^{\infty}\left(\mathcal{F}_{B V}\right), \cdot,\{\cdot, \cdot\}_{\Omega_{B V}}, \Delta_{\mu}\right)$ into a BV algebra [21].

\footnotetext{
${ }^{6} Q$ is essentially the derivation $d_{C}$ interpreted as a vector field.
} 
Remark 4. Theorem 2 is stated for finite-dimensional manifolds. In this case, $\Delta_{\mu}$ always exists. On infinite-dimensional manifolds, a number of complications arise. One needs an appropriate regularisation of $\Delta_{\mu}$, and the corresponding adaptations of statements in Theorem 2 must be checked. In this paper, we are interested in abelian $B F$ theory, which is a noninteracting topological field theory whose partition function (cf. Eq. (2)) is expected to be independent of the gauge fixing, and has been computed to be the Reidermeister (or equivalently) analytic torsion of the de Rham complex [56,57]. More recent work generalised the BV theorem for certain classes of field theories (and gauge fixings) $[22,27]$, while a perturbative approach has been shown to work for the theory at hand in the presence of boundaries [25], and with a regularisation coming from cellular decompositions in [26].

Remark 5. Notice that the notion of Lagrangian submanifold has to be appropriately adapted in infinite dimensions and when dealing with $\mathbb{Z}$-grading. The Lagrangian submanifolds $L$ we will consider in this paper are such that, locally, the symplectic space looks like $L \oplus K$, with the symplectic form given by a nondegenerate pairing between $L$ and $K$. This notion of a Lagrangian submanifold coincides with the one used in [18]. Often $L$ can be seen as the vanishing locus of a Poisson subalgebra $\mathcal{I}$ of the Poisson algebra of functions on $\mathcal{F}_{B F}$, which is also isotropic, i.e. $\left.\Omega_{B F}\right|_{L}=0$. This means that $L$ is isotropic and coisotropic. ${ }^{7}$ For a more in-depth analysis of Lagrangian submanifolds in infinite dimensions and the symplectic category, we refer to [64], building on [63].

Remark 6. For concreteness, in what follows we will discuss field theories where fields are given by differential forms on a manifold. If needed, one can think of the space of fields as a Fréchet vector space, but indeed this specification will not be necessary for our purposes.

\subsection{Partition Functions}

If we look at the quadratic part of the action functional, we can interpret the partition function as a (formal) Gaussian integral. We assume from now on that the action functional is at most quadratic.

In finite dimensions, the result of said integral would be the determinant of the operator featured in the action functional $S_{M}$. In infinite dimensions, this requires defining an appropriate regularisation of determinants.

The standard approach to partition functions for degenerate quadratic functionals follows from Schwarz [56,57], where the resolution of (the kernel of) an elliptic differential operator on a closed Riemannian manifold is presented, which outputs a (co)chain complex, and the partition function is given in terms of products of (regularised) determinants of operators associated with the resolving complex. The explicit example for BF theory is given in Sect. 4.1. In short, the insights from Schwarz allow us to interpret partition functions of quadratic degenerate functionals as (a product of) regularised determinants,

\footnotetext{
${ }^{7}$ This notion coincides with requiring $L$ to be maximal isotropic.
} 
provided a suitable resolution can be found such that the regularised determinants of the associated operators exist.

For the purposes of this paper, instead of the standard zeta function regularisation, we will use the notion of a flat determinant, based on flat traces, inspired by Atiyah and Bott's constructions [2,3]. Details on the definition of flat traces and determinant can be found in several places: [17, Definition 3.12], a microlocal version in [33, Section 2.4], and a mollifier approach in [7, Section 3.2.2]. Since we will not be concerned with the microlocal analysis of operators, we will avoid discussing the necessary tools to define flat traces, and will only work up to the requirements that they exist for the operators we will consider. In this spirit, we give the following definition.

Definition 7. Let $A: V \rightarrow V$ be an operator on an appropriate inner product space, such that the flat trace $\operatorname{tr}^{b}(\exp (-t(A+\lambda)))$ exists for $\lambda \in \mathbb{C}$. We define the flat determinant of $A+\lambda$ to be

$$
\log \operatorname{det}^{b}(A+\lambda):=-\left.\frac{d}{\mathrm{~d} s}\right|_{s=0}\left[\frac{1}{\Gamma(s)} \int_{0}^{\infty} t^{s-1} \operatorname{tr}^{b}\left(\exp (-t(A+\lambda))-\Pi_{\lambda}\right) \mathrm{d} t\right]
$$

where $\Pi_{\lambda}$ is the spectral projector on the kernel of $(A+\lambda)$, whenever the integrals converge.

Remark 8. Observe that if $A$ is such that $\mathrm{e}^{-t(A+\lambda)}$ is trace class, then $\operatorname{tr}\left(\mathrm{e}^{-t(A+\lambda)}\right)=\operatorname{tr}^{b}\left(\mathrm{e}^{-t(A+\lambda)}\right)$. As a consequence, if the zeta-regularised determinant of $A+\lambda$ exists, it coincides with the flat determinant: $\operatorname{det}^{b}(A+\lambda)=$ $\operatorname{det}(A+\lambda)$. See, for example, [7, Proposition 6.8] for details.

Let $A=\operatorname{diag}(B, C)$ be a graded, degree-preserving (block-diagonal) linear map on a finite-dimensional graded vector space. A graded Gaussian integral for $\exp (-\langle y, A x\rangle)$ returns $\operatorname{sdet}(A)^{-1}=\frac{\operatorname{det}(C)}{\operatorname{det}(B)}$. More generally, if $A_{k}$ is the $k$ th component of $A$ acting on a graded space with a finite number of nonzero components, each $A_{k}$ acting on vectors of degree $k$, we get ${ }^{8}$

$$
\operatorname{sdet}(A)=\prod_{k=0}^{n} \operatorname{det}(A)^{(-1)^{k}}
$$

For an introduction to Berezinians and odd integration, see, for example, [51, Section 3.8] and [62], while the original notion was introduced in [10,11].

In infinite dimensions, to an operator $A$ on a graded space we can associate a regularised superdeterminant in the same way, but replacing the determinants on the block operators with their flat-regularised versions. We will use this notion to define the partition function of a degenerate quadratic functional, as follows:

\footnotetext{
${ }^{8}$ Observe that we are considering parities modulo 2. In principle, a graded determinant would return $\prod_{k=0}^{n} \operatorname{det}(A)^{k(-1)^{k}}$. We will not make such a distinction in what follows.
} 
Definition 9. Let $S: V \rightarrow \mathbb{R}$ of the form $S=\frac{1}{2} \int_{M}(x, A x)$ for some operator $A: V \rightarrow V$, with $V$ a (possibly graded) vector space endowed with an inner product $(\cdot, \cdot)$. We define the partition function $Z$ of $S$ to be the square root of the flat (super)determinant of the (graded) operator $A$ :

$$
Z(S)=\int_{V} \mathrm{e}^{i S_{M}}:=\left|\operatorname{sdet}^{b}(A)\right|^{-\frac{1}{2}} .
$$

When the field theory has symmetries, we assume that $V$ is further endowed with a $(-1)$ symplectic form. A choice of a Lagrangian submanifold $\mathbb{L} \subset V$ will be called gauge fixing, and we define the partition function of $S$ in the gauge fixing $\mathbb{L}$ to be

$$
Z(S, \mathbb{L}):=Z\left(\left.S\right|_{\mathbb{L}}\right)
$$

Remark 10. To obtain a true generalisation of finite-dimensional Gaussian integrals, one should append to formula (12) the phase factor $\exp \left(-i \frac{\pi}{4} \operatorname{sg}(A)\right)$ where $\operatorname{sg}(A)$ is the signature of the operator $A$, appropriately regularised. Since in what follows we will not discuss the phase of partition functions, we will omit this term from the definition.

Remark 11. Observe that often one encounters the situation in which $S_{M}=$ $\int_{M}(y, B x)$ for some operator $B$ and $x, y \in V^{\prime}$ for some space $V^{\prime}$. Define ${ }^{9}$ $V=V^{\prime} \oplus V^{\prime}$ and

$$
A=\left(\begin{array}{cc}
0 & B \\
B^{t} & 0
\end{array}\right)
$$

so that $(y, B x)=\frac{1}{2}(z, A z)$ with $z=(x, y) \in V$. Then $Z=\left|\operatorname{sdet}^{b}(A)\right|^{-\frac{1}{2}}=$ $\left|\operatorname{sdet}^{b}(B)\right|^{-1}$.

Remark 12. Let $V$ be a graded vector space and $V[k]$ its $k$-shift, so that $(V[k])^{i}:=V^{i+k}$. In particular, if $z \in V[1]$ is a homogeneous element of degree $k$ it will be parametrised by homogeneous elements in $V$ of opposite parity. In particular, if $A$ is a graded linear map on $V$, the Gaussian integral

$$
\int_{V[1]} \mathrm{e}^{-\frac{1}{2}(z, A, z)}:=\left|\operatorname{sdet}^{b}(A)\right|^{\frac{1}{2}} .
$$

\section{Geometric Setting}

This section establishes the geometry and notations. In the following three sections, we progressively introduce more structure to our initial set-up of a flat vector bundle over a manifold, whose twisted cohomology is trivial. The plainest setting involves a differentiable manifold endowed with a flat vector bundle. On top of that, we consider the introduction of either a Riemannian structure or a contact structure. The intersection of the two will require the base manifold to display Anosov dynamics.

\footnotetext{
${ }^{9}$ Note that the $V^{\prime}$ components in $V$ are not considered to have different degrees, i.e. $A$ is an even matrix of graded operators.
} 
It is useful to distinguish between these geometric settings as when we will only need certain geometric properties when discussing different field theories. This distinction between geometric data with which a differentiable manifold is endowed reflects the practice of complementing topological theories with additional geometric structures (e.g. Riemannian or contact) for the sake of gauge fixing.

\subsection{Flat Vector Bundle}

Let $M$ be an $N$-dimensional compact manifold without boundary which is oriented and connected. Let $\rho: \pi_{1}(M) \rightarrow U\left(\mathbb{C}^{r}\right)$ denote a unitary representation. This representation endows $M$ with a Hermitian vector bundle $(E, h)$ of rank $r$ with flat connection $\nabla$. We collectively denote these data by $(M, E)$.

Let $\Omega^{\bullet}(M ; E)$ denote the space of (smooth) differential forms on $M$ taking values in $E$, and let

$$
d_{\nabla} \equiv d_{k}: \Omega^{k}(M, E) \rightarrow \Omega^{k+1}(M, E)
$$

be two notations for the twisted de Rham differential. We denote by $H^{\bullet}(M ; E)$ the cohomology associated with the twisted de Rham complex $\left(\Omega^{\bullet}(M ; E), d_{\nabla}\right)$, with Betti numbers $\beta_{k}:=\operatorname{dim} H^{k}(M ; E)$.

From now on, we will assume that $(M, E)$ is such that its twisted de Rham complex is acyclic, i.e. $\beta_{k}=0$ for all $0 \leq k \leq N$.

\subsection{Riemannian Structure and Analytic Torsion}

Suppose $(M, E)$ is supplemented with a Riemannian metric $g_{M}$. We collectively denote these data $\left(M, E, g_{M}\right)$. Let $\mathrm{dVol}$ denote the associated volume form and let $\langle\cdot, \cdot\rangle$ be the inherited inner product on $\bigwedge^{\bullet} T^{*} M$. The Hodge star $\star$ : $\bigwedge^{k} T^{*} M \rightarrow \bigwedge^{N-k} T^{*} M$ is defined through

$$
u \wedge \star v=\langle u, v\rangle \mathrm{d} \operatorname{Vol}
$$

for $u, v \in \bigwedge^{k} T^{*} M$. This lifts to $E$-valued forms by identifying $E$ with its dual via the Hermitian metric $h$. An inner product is then placed on $\Omega^{\bullet}(M ; E)$ by declaring

$$
(u, v)=\int_{M}[u \wedge \star v]^{\text {top }}
$$

for $u, v \in \Omega^{\bullet}(M ; E)$ with top refers to only taking the top-form (degree $N$ ) part of $u \wedge \star v$.

The de Rham differential has an adjoint

$$
d_{\nabla}^{*} \equiv d_{k}^{*}: \Omega^{k+1}(M, E) \rightarrow \Omega^{k}(M, E)
$$

which provides the twisted Laplace-de Rham operator (henceforth simply Laplacian)

$$
\Delta_{k}:=\left(d_{\nabla}^{*}+d_{\nabla}\right)^{2}: \Omega^{k}(M ; E) \rightarrow \Omega^{k}(M ; E) .
$$

When acting on $L^{2}\left(M ; \bigwedge^{k} T^{*} M \otimes E\right)$, the Laplacian has nonnegative eigenvalues $\lambda_{n} \geq 0$. Denote by $\Pi_{\lambda}$ the $L^{2}$ spectral projector onto the kernel of $\Delta_{k}+\lambda$, and consider the function 


$$
F_{\Delta_{k}}(\lambda, s):=\frac{1}{\Gamma(s)} \int_{0}^{\infty} t^{s-1} \operatorname{tr}^{b}\left(\mathrm{e}^{-t\left(\Delta_{k}+\lambda\right)}-\Pi_{\lambda}\right) \mathrm{d} t .
$$

The (flat)-regularised determinant of the operator $\Delta_{k}+\lambda$ is

$$
\log \operatorname{det}^{b}\left(\Delta_{k}+\lambda\right)=-\left.\frac{\partial}{\partial s}\right|_{s=0} F_{\Delta_{k}}(\lambda, s) .
$$

We also write $f_{\Delta_{k}}(s):=F_{\Delta_{k}}(0, s)$ and observe that $f_{\Delta_{k}}(s)=\sum_{\lambda_{j}>0} \lambda_{j}{ }^{-s}$.

Definition 13. The analytic torsion of $M[53]$ is defined to be

$$
\tau_{\rho}(M):=\prod_{k=1}^{N} \operatorname{det}^{b}\left(\Delta_{k}\right)^{\frac{k}{2}(-1)^{k+1}} .
$$

Alternatively, we can write

$$
2 \log \tau_{\rho}(M)=\left.\sum_{k=1}^{N}(-1)^{k} k \frac{d}{\mathrm{~d} s}\right|_{s=0} f_{\Delta_{k}}(s) .
$$

The Hodge decomposition provides an orthogonal decomposition of $L^{2}(M$; $\bigwedge^{\bullet} T^{*} M \otimes E$ ) into exact and coexact forms. (No harmonic forms are present due to acyclicity.) Introducing $d_{k}^{*} d_{k}:=\left.\Delta_{k}\right|_{\text {coexact }}$, we can also write the analytic torsion as [57] (see also $[26,50]$ )

$$
\tau_{\rho}(M)=\prod_{k=0}^{N-1} \operatorname{det}^{b}\left(d_{k}^{*} d_{k}\right)^{\frac{1}{2}(-1)^{k}},
$$

which alternatively reads

$$
2 \log \tau_{\rho}(M)=\left.\sum_{k=0}^{N-1}(-1)^{k+1} \frac{d}{\mathrm{~d} s}\right|_{s=0} f_{d_{k}^{*} d_{k}}(s) .
$$

\subsection{Contact Structure}

Suppose that $(M, E)$ is supplemented with a contact form $\alpha \in \Omega^{1}(M)$, and $\operatorname{dim}(M)=N=2 n+1$. We will denote $\mathrm{dVol}=\alpha \wedge(\mathrm{d} \alpha)^{n}$. Let $X$ be the associated Reeb vector field, defined by the relations

$$
\iota_{X} \alpha=1, \quad \iota_{X} \mathrm{~d} \alpha=0 .
$$

We collectively denote these data $(M, E, X)$.

Denote by $T_{0}^{*} M$ the $2 n$-rank subbundle of $T^{*} M$ defined as the conormal of $X$, so that pointwise $T^{*} M=\mathbb{R} \alpha+T_{0}^{*} M$. Transferring this to the space of $E$-valued differential forms, we write

$$
\Omega^{k}(M, E)=\Omega_{0}^{k}(M, E) \oplus \alpha \wedge \Omega_{0}^{k-1}(M, E),
$$

where $\Omega_{0}^{k}(M, E)=\left.\operatorname{ker} \iota_{X}\right|_{\Omega^{k}(M)}$. 
2.3.1. Contact Riemannian Structure. Let $(M, E, X)$ as above and introduce a metric $g_{M}$ on $M$ of the form $g_{M}=\alpha^{2}+g_{0}$ such that $T^{*} M=\mathbb{R} \alpha+T_{0}^{*} M$ becomes an orthogonal decomposition. Let $\star$ and $\star_{0}$ denote the Hodge stars associated with $\left(T^{*} M, g_{M}\right)$ and $\left(T_{0}^{*} M, g_{0}\right)$, respectively. We may choose $g_{0}$ such that $\star \alpha=(\mathrm{d} \alpha)^{n}=\star_{0} 1$ whence the Hodge star behaves nicely with respect to the splitting of $T^{*} M$. Specifically, we have maps

$$
\begin{aligned}
\star: \Omega_{0}^{k}(M, E) & \rightarrow \alpha \wedge \Omega_{0}^{N-k-1}(M, E) & \star: \alpha \wedge \Omega_{0}^{k}(M, E) \rightarrow \Omega_{0}^{N-k-1}(M, E) \\
\varphi & \mapsto(-1)^{k} \alpha \wedge \star_{0} \varphi & \alpha \wedge \psi \mapsto \star_{0} \psi
\end{aligned}
$$

Moreover, noting that $\langle\alpha \wedge \varphi, \alpha \wedge \varphi\rangle=\langle\varphi, \varphi\rangle$ on $\Omega_{0}^{\bullet}(M, E)$ we conclude $\left(\iota_{X}\right)^{T}=$ $\alpha \wedge$. When a metric is chosen in this way, compatible with the contact structure, we collectively denote these data $\left(M, E, X, g_{M}\right)$.

Definition 14. Considering the maps

$$
\alpha \wedge: \Omega_{0}^{\bullet}(M, E) \rightarrow \alpha \wedge \Omega_{0}^{\bullet}(M, E), \quad \iota_{X}: \alpha \wedge \Omega_{0}^{\bullet}(M, E) \rightarrow \Omega_{0}^{\bullet}(M, E),
$$

we set

$$
\operatorname{sdet}^{b}(\alpha \wedge)=\operatorname{sdet}^{b}\left(\iota_{X}\right):=\left|\operatorname{sdet}^{b}\left(\iota_{X} \circ \alpha \wedge\right)^{\frac{1}{2}}\right|=1 .
$$

\subsection{Anosov Dynamics}

Suppose $M$ is supplemented with a flow $\varphi_{t}: M \rightarrow M$ for $t \in \mathbb{R}$. We will reuse $X \in C^{\infty}(M ; T M)$ to denote the vector field which generates $\varphi_{t}$.

Definition 15. The flow is Anosov if there exists a $\mathrm{d} \varphi_{t}$-invariant continuous splitting of the tangent bundle:

$$
T_{x} M=E_{n}(x) \oplus E_{s}(x) \oplus E_{u}(x), \quad E_{n}(x)=\mathbb{R} X_{x},
$$

and for a given norm $\|\cdot\|$ on $T M$, there exist constants $C, \lambda>0$ so that for all $t \geq 0$,

$$
\begin{aligned}
& \forall v \in E_{s}(x), \quad\left\|\mathrm{d} \varphi_{t}(x) v\right\| \leq C \mathrm{e}^{-\lambda t}\|v\|, \\
& \forall v \in E_{u}(x), \quad\left\|\mathrm{d} \varphi_{-t}(x) v\right\| \leq C \mathrm{e}^{-\lambda t}\|v\| .
\end{aligned}
$$

The subbundles $E_{n}, E_{s}$ and $E_{u}$ are, respectively, called neutral, stable and unstable.

Remark 16. We will prefer to work with the cotangent bundle $T^{*} M$ due to the Lie derivative acting naturally on differential forms. The cotangent bundle also has a decomposition $T_{x}^{*} M=E_{n}^{*}(x) \oplus E_{s}^{*}(x) \oplus E_{u}^{*}(x)$ whose stable and unstable bundles are understood through the action of $\left(\mathrm{d} \varphi_{-t}\right)^{T}$ (rather than $\left.\mathrm{d} \varphi_{t}\right)$.

Henceforth, we will always assume that the stable and unstable bundles are orientable and each have rank $n$. 
2.4.1. A Guiding Example. We provide an example of the geometric setting discussed in the previous subsections. Let $(\Sigma, g)$ be a compact manifold without boundary which is oriented, connected and of dimension $n+1$. Suppose that $\Sigma$ has sectional curvature which is everywhere strictly negative. Let $M:=S_{g}^{*} \Sigma$ be the unit cotangent bundle of $\Sigma$. Set $\alpha \in \Omega^{1}(M)$ to be the pullback of the canonical one form on $T^{*} \Sigma$. Then $(M, \alpha)$ is a contact manifold, and the Reeb vector field $X_{g} \in C^{\infty}(M ; T M)$ generates the geodesic flow $\varphi_{t}$ which is Anosov $[1,5,6]$.

If we consider $M=S_{g}^{*} \Sigma$ within the geometric setting $(M, E)$ of Sect. 2.1 (in particular the representation $\rho$ is unitary and $\nabla$ is flat), we denote the resulting contact, Anosov, Riemannian data on $S_{g}^{*} \Sigma$ by

$$
\left(M=S_{g}^{*} \Sigma, E, X_{g}, g\right) .
$$

Remark 17. $M \rightarrow \Sigma$ is an $\mathbb{S}^{n}$-bundle, and if $n \geq 2$, then $\pi_{1}\left(\mathbb{S}^{n}\right)=0$ whence representations of $\pi_{1}(\Sigma)$ are in one-to-one correspondence with representations of $\pi_{1}(M)$.

Remark 18. Observe that in the case of a unitary representation and a flat vector bundle, and when $n \geq 2$, the preceding remark implies that $\tau_{\rho}(M)=$ $\left(\tau_{\rho}(\Sigma)\right)^{2}[37$, Section 1, p. 526]. For the rest of this article, particularly the announcements of theorems and conjectures, we have chosen to emphasise the role of $M=S_{g}^{*} \Sigma$ rather than $\Sigma$.

\section{Ruelle Zeta Function}

Consider the geometric data $(M, E, X)$ of Sect. 2.3 and assume the flow $\varphi_{t}$ associated with $X$ is Anosov (see Sect. 2.4). We denote by $\mathcal{P}$ the set of primitive orbits of the flow $\varphi_{t}$ and by $\ell(\gamma)$ the period of any given $\gamma \in \mathcal{P}$.

Definition 19. The Ruelle zeta function (associated with the trivial representation of $\left.\pi_{1}(M)\right)$ is defined as

$$
\zeta(\lambda):=\prod_{\gamma \in \mathcal{P}}\left(1-\mathrm{e}^{-\lambda \ell(\gamma)}\right)
$$

whose convergence is assured for $\operatorname{Re} \lambda \gg 1$. The Ruelle zeta function twisted by an arbitrary representation $\rho$ is

$$
\zeta_{\rho}(\lambda):=\prod_{\gamma \in \mathcal{P}} \operatorname{det}\left(I-\rho([\gamma]) \mathrm{e}^{-\lambda \ell(\gamma)}\right),
$$

whose convergence is again assured for $\operatorname{Re} \lambda \gg 1$.

Here, $[\gamma]$ represents the conjugacy class of $\gamma$ in $\pi_{1}(M)$. It has been shown that the zeta functions continue meromorphically to $\mathbb{C}[12,33,41,48]$. We have the following: 
Theorem 20 ([37]). Let $(M, E, X, g)$ be the geometric data of Sect. 2.4.1, where $M=S_{g}^{*} \Sigma$, with $\Sigma$ a closed, oriented hyperbolic manifold $\Sigma=\Gamma \backslash \mathbb{H}^{n+1}$, and $g$ the induced hyperbolic metric. Then, the Ruelle zeta function, defined for $\operatorname{Re}(s)>n$ by Eq. (34), extends meromorphically to $\mathbb{C}$ and

$$
\left|\zeta_{\rho}(0)\right|^{(-1)^{n}}=\tau_{\rho}(M)
$$

This fact has inspired a conjecture [38]:

Conjecture 21 (Fried). Let $(M, E, X, g)$ be the geometric data of Sect. 2.4.1 with $\operatorname{dim}(M)=2 n+1$. Then the (twisted) Ruelle zeta function computes the analytic torsion:

$$
\left|\zeta_{\rho}(0)\right|^{(-1)^{n}}=\tau_{\rho}(M)
$$

\subsection{Differential Forms Decomposition}

This section shows how the (twisted) Ruelle zeta function may be written as an alternating product of zeta functions associated with $\left(\wedge^{k} T_{0}^{*} M\right) \otimes E$ for $0 \leq k \leq 2 n$.

Given a closed orbit $\gamma$, of length $\ell(\gamma)$ and a point $x \in M$ in the orbit, let $P(\gamma, x)$ denote the linearised Poincaré map on the fibre of $T_{0}^{*} M$ above $x$ :

$$
P(\gamma, p):=\left(\mathrm{d} \varphi_{-\ell(\gamma)}\right)^{T}: T_{0}^{*} M_{(x)} \rightarrow T_{0}^{*} M_{(x)}
$$

This map is conjugate to $P\left(\gamma, x^{\prime}\right)$ for other $x^{\prime}$ in the same orbit $\gamma$, and as we need only evaluate this map's trace and determinant, we will refer to all maps as $P(\gamma)$. Recall $T_{0}^{*} M$ is the subbundle of $T^{*} M$ conormal to the vector field $X$.

We start with the basic linear algebra identity

$$
\operatorname{det}(I-A)=\sum_{k=0}^{m}(-1)^{k} \operatorname{tr}\left(\wedge^{k} A\right)
$$

for an endomorphism $A$ on an $m$-dimensional vector space and the observation that $P(\gamma)$ has precisely $n$ eigenvalues greater than 1 (where $n$ is the rank of $\left.E_{u}^{*}\right)$. Therefore, for $j \geq 1$

$$
(-1)^{n}\left|\operatorname{det}\left(I-P(\gamma)^{j}\right)\right|=\sum_{k=0}^{2 n}(-1)^{k} \operatorname{tr}\left(\wedge^{k} P(\gamma)^{j}\right)
$$


Using identity (37) to obtain equality (38c), we derive

$$
\begin{aligned}
\log \zeta_{\rho}(\lambda) & =\sum_{\gamma \in \mathcal{P}} \operatorname{tr} \log \left(I-\rho([\gamma]) \mathrm{e}^{-\lambda \ell(\gamma)}\right) \\
& =-\sum_{\gamma \in \mathcal{P}} \operatorname{tr} \sum_{j=1}^{\infty} \frac{1}{j} \mathrm{e}^{-\lambda j \ell(\gamma)} \rho([\gamma])^{j} \\
& =-\sum_{\gamma \in \mathcal{P}} \sum_{j=1}^{\infty} \frac{1}{j} \mathrm{e}^{-\lambda j \ell(\gamma)} \operatorname{tr}\left(\rho([\gamma])^{j}\right)\left((-1)^{n} \sum_{k=0}^{2 n} \frac{(-1)^{k} \operatorname{tr}\left(\wedge^{k} P(\gamma)^{j}\right)}{\left|\operatorname{det}\left(I-P(\gamma)^{j}\right)\right|}\right) \\
& =(-1)^{n} \sum_{k=0}^{2 n}(-1)^{k} \log \zeta_{\rho, k}(\lambda) .
\end{aligned}
$$

Equation (38d) in the preceding display defines implicitly the Ruelle zeta function twisted by $\rho$ upon restriction to $k$-forms

$$
\zeta_{\rho, k}(\lambda):=\exp \left(-\sum_{\gamma \in \mathcal{P}} \sum_{j=1}^{\infty} \frac{1}{j} \frac{\mathrm{e}^{-\lambda j \ell(\gamma)} \operatorname{tr}\left(\rho([\gamma])^{j}\right) \operatorname{tr}\left(\wedge^{k} P(\gamma)^{j}\right)}{\left|\operatorname{det}\left(I-P(\gamma)^{j}\right)\right|}\right)
$$

so in the form of a compact equality, we have

$$
\zeta_{\rho}(\lambda)^{(-1)^{n}}=\prod_{k=0}^{2 n} \zeta_{\rho, k}(\lambda)^{(-1)^{k}} .
$$

\subsection{Ruelle Zeta Function as Regularised Determinant}

We aim to link the function $\zeta_{\rho, k}$ with the operator $\mathcal{L}_{X, k}$ acting on $\Omega_{0}^{k}(M ; E)$. This is done with the help of the Atiyah-Bott-Guillemin trace formula [42]. We use the notation

$$
\mathrm{e}^{-t \mathcal{L}_{X, k}}=\varphi_{-t}^{*}: L^{2}\left(M ; \bigwedge^{k} T_{0}^{*} M \otimes E\right) \rightarrow L^{2}\left(M ; \bigwedge^{k} T_{0}^{*} M \otimes E\right) .
$$

and write the Schwartz kernel $K_{k}(t, \cdot, \cdot)$ such that

$$
\left(\mathrm{e}^{-t \mathcal{L}_{X, k}} \psi\right)(x)=\int_{M} K_{k}(t, x, y) \psi(y) \mathrm{d} y .
$$

Due to the microlocal structure of $K_{k}$ we may take its flat trace, which leads to the Atiyah-Bott-Guillemin trace formula:

$$
\operatorname{tr}^{b} \mathrm{e}^{-t \mathcal{L}_{X, k}}=\sum_{\gamma \in \mathcal{P}} \sum_{j=1}^{\infty} \ell(\gamma) \delta(t-j \ell(\gamma)) \frac{\operatorname{tr}\left(\rho([\gamma])^{j}\right) \operatorname{tr}\left(\wedge^{k} P(\gamma)^{j}\right)}{\left|\operatorname{det}\left(I-P(\gamma)^{j}\right)\right|}
$$

as a distribution on $\mathbb{R}_{+}$. Integrating this distribution against the function $-t^{-1} \mathrm{e}^{-t \lambda}$ provides an integral representation of $\zeta_{\rho, k}(\lambda)$ :

$$
\log \zeta_{\rho, k}(\lambda)=-\int_{0}^{\infty} t^{-1} \mathrm{e}^{-t \lambda} \operatorname{tr}^{b} \mathrm{e}^{-t \mathcal{L}_{X, k}} \mathrm{~d} t .
$$


Consider now the following function, dependent on two variables:

$$
F_{\mathcal{L}_{X, k}}(\lambda, s):=\frac{1}{\Gamma(s)} \int_{0}^{\infty} t^{s-1} \operatorname{tr}^{b} \mathrm{e}^{-t\left(\mathcal{L}_{X, k}+\lambda\right)} \mathrm{d} t .
$$

This function is holomorphic for small $s$ (near $s=0$ ) and for $\operatorname{Re}(\lambda) \gg 1$ (see Sect. 3.3). Naively, at $s=0$ the integrand poses a problem due to the $t^{-1}$ structure as $t \rightarrow 0$; however, the trace formula (Eq. (43)) provides a natural cut-off in small $t$ so that we avoid this problem. Moreover, for small $s$ we expand $1 / \Gamma(s)=s+O\left(s^{2}\right)$ showing

$$
\begin{aligned}
\left.\partial_{s}\right|_{s=0} F_{\mathcal{L}_{X, k}}(\lambda, s) & =\left.\left(\left.\partial_{s}\right|_{s=0} \frac{1}{\Gamma(s)}\right) \cdot \int_{0}^{\infty} t^{s-1} \operatorname{tr}^{b} \mathrm{e}^{-t\left(\mathcal{L}_{X, k}+\lambda\right)} \mathrm{d} t\right|_{s=0} \\
& =-\log \zeta_{\rho, k}(\lambda) .
\end{aligned}
$$

Recalling Definition 7 for the flat determinant of an operator now indicates that, for $\operatorname{Re} \lambda \gg 1$

$$
\log \operatorname{det}^{b}\left(\mathcal{L}_{X, k}+\lambda\right) \equiv-\left.\partial_{s}\right|_{s=0} F_{\mathcal{L}_{X, k}}(\lambda, s)=\log \zeta_{\rho, k}(\lambda) .
$$

Since $\zeta_{\rho, k}(\lambda)$ has a meromorphic extension to the complex plane, so does the function $\operatorname{det}^{b}\left(\mathcal{L}_{X, k}+\lambda\right)$. Assuming there are no poles at $\lambda=0$, we sensibly have $\operatorname{det}^{b}\left(\mathcal{L}_{X, k}\right)$ as the value at zero of the meromorphic extension of the Ruelle zeta function (restricted to $k$-forms) at zero:

$$
\operatorname{det}^{b}\left(\mathcal{L}_{X, k}\right)=\zeta_{\rho, k}(0) .
$$

The decomposition in Eq. (40) of the Ruelle zeta function now gives an operator interpretation of the Ruelle zeta function:

$$
\zeta_{\rho}(\lambda)^{(-1)^{n}}=\prod_{k=0}^{2 n} \operatorname{det}^{b}\left(\mathcal{L}_{X, k}+\lambda\right)^{(-1)^{k}} .
$$

Remark 22. Notice that, due to the decomposition (40) and the trace formula (43), we can consider $\zeta_{\rho}$ as directly dependent on an Anosov vector field $X$. To stress this fact, we will use the notation $\zeta(X, \lambda)\left(\operatorname{resp} . \zeta_{k}(X, \lambda)\right)$ instead of $\zeta_{\rho}(\lambda)\left(\operatorname{resp} . \zeta_{\rho, k}(\lambda)\right)$.

\subsection{Meromorphic Extension of the Resolvent}

In the preceding section, we related the resolvent $\left(\mathcal{L}_{X, k}+\lambda\right)^{-1}$ to $\zeta_{\rho, k}(\lambda)$ for $\lambda \gg 1$. Here, we announce a more precise statement for the meromorphic extension of $\left(\mathcal{L}_{X, k}+\lambda\right)^{-1}[33]$.

The operator norm of $\mathrm{e}^{-t \mathcal{L}_{X, k}}$ is bounded by $\mathrm{e}^{C_{0} t}$ for some $C_{0}>0$. Therefore, the resolvent $\left(\mathcal{L}_{X, k}+\lambda\right)^{-1}$, as an operator on $L^{2}$ sections, exists for $\operatorname{Re} \lambda>C_{0}$ and is given by the formula

$$
\left(\mathcal{L}_{X, k}+\lambda\right)^{-1}=\int_{0}^{\infty} \mathrm{e}^{-t\left(\mathcal{L}_{X, k}+\lambda\right)} \mathrm{d} t
$$

The restricted resolvent

$$
R_{k}(\lambda)=\left(\mathcal{L}_{X, k}+\lambda\right)^{-1}: C^{\infty}\left(M ; \bigwedge^{k} T_{0}^{*} M \otimes E\right) \rightarrow \mathcal{D}^{\prime}\left(M ; \bigwedge^{k} T_{0}^{*} M \otimes E\right)
$$


has a nowhere-vanishing meromorphic continuation to $\mathbb{C}$ whose poles are of finite rank, and are called Pollicott-Ruelle resonances. For each $\lambda_{0} \in \mathbb{C}$, we have the expansion

$$
R_{k}(\lambda)=R_{k}^{H}(\lambda)+\sum_{j=1}^{J\left(\lambda_{0}\right)} \frac{(-1)^{j-1}\left(\mathcal{L}_{X, k}+\lambda_{0}\right)^{j-1} \Pi_{\lambda_{0}}}{\left(\lambda-\lambda_{0}\right)^{j}}
$$

where $R_{k}^{H}$ is holomorphic near $\lambda_{0}$ and $\Pi_{\lambda_{0}}: \quad C^{\infty}$ $\left(M ; \bigwedge^{k} T_{0}^{*} M \otimes E\right) \rightarrow \mathcal{D}^{\prime}\left(M ; \bigwedge^{k} T_{0}^{*} M \otimes E\right)$ is a finite rank projector. The range of $\Pi_{\lambda_{0}}$ defines (generalised) resonant states. They are characterised as

Range $\Pi_{\lambda_{0}}=\left\{\varphi \in \mathcal{D}^{\prime}\left(M, \bigwedge^{k} T_{0}^{*} M \otimes E\right): \mathrm{WF}(u) \subset E_{u}^{*},\left(\mathcal{L}_{X, k}+\lambda_{0}\right)^{J\left(\lambda_{0}\right)} \varphi=0\right\}$,

where WF refers to the wave front of a distribution (or current), $E_{u}^{*}$ is the unstable bundle referenced in Remark 16 and $J\left(\lambda_{0}\right)$ denotes the multiplicity. The adjective "generalised" refers to the possibility that the pole may not be simple (and is superfluous in the case $J\left(\lambda_{0}\right)=1$ ).

Finally, the poles of the meromorphic continuation $R_{k}(\lambda)$ correspond to zeros of the zeta function $\zeta_{k}(\lambda)$ (which is entire for each $k$ ), and the rank of the projector $\Pi_{\lambda}$ equals the multiplicity of the zero.

\section{BF Theory on Contact Manifolds}

In this section, we will analyse a field theory that goes under the name of $B F$ theory, ${ }^{10}$ in the Batalin-Vilkovisky (BV) formalism. Consider the basic geometric data $(M, E)$ of Sect. 2.1. The classical version of abelian $B F$ theory (i.e. without BV Formalism) is given by the following assignment:

Definition 23. Define the space of classical fields to be $F_{B F}:=\Omega^{1}(M, E) \oplus$ $\Omega^{N-2}(M, E)$, and the BF action functional:

$$
S_{B F}=\int_{M} B \wedge d_{\nabla} A
$$

Then we call $B F$ theory the assignment $(M, E) \rightsquigarrow\left(F_{B F}, S_{B F}\right)$.

Remark 24. Recall that Sect. 2.1 equips $E$ with a Hermitian metric $h$. This metric has been implicitly used to couple $B$ and $A$. Specifically, both $B$ and $d_{\nabla} A$ take values in $E$ whence $h$ must be used so that $B \wedge d_{\nabla} A \in \Omega^{N}(M)$.

\footnotetext{
${ }^{10}$ The name $B F$ comes from the tradition of denoting fields with $B$ and $A$, and the Lagrangian density with $B \wedge F_{A}$.
} 
Remark 25. It is easy to see that shifting either $B$ or $A$ by a $d_{\nabla}$-exact form leaves the action functional unchanged. ${ }^{11}$ This goes under the name of reducible symmetry, and it is conveniently treated by means of the BV formalism.

Let us consider the space of differential forms $\Omega^{-\bullet}(M, E)$ as a $\mathbb{Z}$-graded vector space, such that homogeneous forms $\omega^{(k)} \in \Omega^{k}(M, E)$ will have degree $\left|\omega^{(k)}\right|:=-k$. We define the space of Batalin-Vilkovisky fields for $B F$ theory to be the graded vector space

$$
\mathcal{F}_{B F}:=\Omega^{-\bullet}(M, E)[1] \oplus \Omega^{-\bullet}(M, E)[N-2] \ni(\mathbb{A}, \mathbb{B})
$$

where the degree shift means that a $k$-form in $\Omega^{-\bullet}(M, E)[1]$ will have degree $|\mathbb{A}|=1-k$. The symplectic structure reads

$$
\Omega_{B F}=\int_{M}[\delta \mathbb{B} \delta \mathbb{A}]^{\text {top }},
$$

and we define an action functional on $\mathcal{F}_{B F}$ as

$$
\mathbb{S}_{B F}=\int_{M}\left[\mathbb{B} d_{\nabla} \mathbb{A}\right]^{\mathrm{top}} .
$$

Observe that $\left|\mathbb{S}_{B F}\right|=0$ and $\left|\Omega_{B F}\right|=-1$.

Remark 26. We stress that, although the functional form of $S_{B F}$ in Eq. (55) and $\mathbb{S}_{B F}$ in (58) is the same, in the latter $\mathbb{B}$ and $\mathbb{A}$ are inhomogeneous forms with an additional shift in degree. Such a degree shift effectively switches the total parity of inhomogeneous forms, so that, if $N$ is odd, even forms will have odd parity and vice versa. This will have a crucial impact in the partition function interpretation of quantities such as the analytic torsion and Ruelle zeta function.

Definition 27. The assignment $(M, E) \rightsquigarrow\left(\mathcal{F}_{B F}, \Omega_{B F}, \mathbb{S}_{B F}, Q_{B F}\right)$, with

$$
Q_{B F} \mathbb{B}=d_{\nabla} \mathbb{B}, \quad Q_{B F} \mathbb{A}=d_{\nabla} \mathbb{A}
$$

is called $B F$ theory in the Batalin-Vilkovisky formalism.

\subsection{Analytic Torsion from Resolutions of de Rham Differential}

In this section, we will discuss the relation of analytic torsion with degenerate action functionals, following Schwarz $[56,57]$. This is related to BF, as we will highlight in what follows. In Sect. 4.2, we will interpret this relation in terms of a gauge fixing for $B F$ theory in the Batalin-Vilkovisky framework.

Consider the geometric data $\left(M, E, g_{M}\right)$ of Sect. 2.2. Note that the only importance of the vector bundle is to ensure acyclicity of the twisted de Rham complex. This requirement will be necessary in Sect. 5; however, what we will say here can be extended to nontrivial cohomology along the lines of $[25,26]$.

\footnotetext{
${ }^{11}$ Strictly speaking this is true only up to boundary terms. We will assume that $M$ has no boundary, but otherwise the analysis of boundary terms is relevant, and can be performed with a version of the BV formalism [25].
} 
We can define the partition function associated with abelian $B F$ theory as follows. We first need a resolution of the kernel of the operator featuring in the action functional, represented then as the 0th cohomology of a chain complex. For abelian $B F$ theory, it is given by the following:

$$
0 \rightarrow V_{N-2} \stackrel{T_{N-2}}{\longrightarrow} V_{N-1} \stackrel{T_{N-1}}{\longrightarrow} \ldots \stackrel{T_{3}}{\longrightarrow} V_{2} \stackrel{T_{2}}{\longrightarrow} V_{1} \stackrel{T_{1}}{\longrightarrow} V_{0} \stackrel{T}{\longrightarrow} V_{0} \rightarrow 0
$$

where

$$
\begin{array}{ll}
V_{0}=\Omega^{1}(M) \oplus \Omega^{N-2}(M), & T=\left[\begin{array}{cc}
0 & \star d_{N-2} \\
\star d_{1} & 0
\end{array}\right] ; \\
V_{1}=\Omega^{0}(M) \oplus \Omega^{N-3}(M), & T_{1}=\left[\begin{array}{cc}
d_{0} & 0 \\
0 & d_{N-3}
\end{array}\right] ; \\
V_{2}=\Omega^{N-4}(M), & T_{2}=\left[\begin{array}{c}
0 \\
d_{N-4}
\end{array}\right] ; \\
V_{k}=\Omega^{N-(k+2)}(M), & T_{k}=d_{N-(k+2)}
\end{array}
$$

for $3 \leq k \leq N-2$. This implies $T_{k} T_{k}^{*}=d_{N-(k+2)} d_{N-(k+2)}^{*}$ and

$$
\begin{aligned}
& T^{2}=\left[\begin{array}{cc}
d_{1}^{*} d_{1} & 0 \\
0 & d_{N-2}^{*} d_{N-2}
\end{array}\right], \quad T_{1} T_{1}^{*}=\left[\begin{array}{cc}
d_{0} d_{0}^{*} & 0 \\
0 & d_{N-3} d_{N-3}^{*}
\end{array}\right], \\
& T_{2} T_{2}^{*}=\left[\begin{array}{lc}
0 & 0 \\
0 & d_{N-4} d_{N-4}^{*}
\end{array}\right] .
\end{aligned}
$$

In fact, denoting $C:=(A, B) \in V_{0}$, the operator $T$ allows us to rewrite

$$
S_{B F}=\int_{M} B \wedge d A=\frac{1}{2}(C, T C)
$$

where $($,$) is the inner product (17)$ on $V_{0}$ as discussed in Sect. 2.2. Then, the partition function of the degenerate action functional (63), with respect to the resolution (60), is defined by Schwarz in [57] to be:

$$
Z_{\mathrm{Sch}}\left[T, T_{i}\right]:=\operatorname{det}^{\mathrm{b}}\left(T^{2}\right)^{-\frac{1}{4}} \prod_{k=1}^{N-2} \operatorname{det}^{\mathrm{b}}\left(T_{k} T_{k}^{*}\right)^{(-1)^{k+1} \frac{1}{2}}
$$

Remark 28. The procedure outlined above recursively produces spaces $V_{k}$ at every stage in order to parametrise the relevant quotients $V_{k} / \operatorname{ker}\left(T_{k}\right)$, on which integration makes sense. Indeed, said quotients coincide with $\operatorname{coker}\left(T_{k+1}\right) \simeq$ $\operatorname{ker}\left(T_{k+1}^{*}\right)$, and the localisation to the correct integration subspaces is obtained by restriction to $\operatorname{ker}\left(T_{k}^{*}\right)$. Thus, Schwarz's definition of the partition function goes through an extension to a larger space of fields, and the choice of a subspace where integration is well defined: the kernel of the map $\mathbb{T}:=\oplus_{j=1}^{N-2} T_{j}^{*}$. Notice that this construction produces a resolution in the sense of Eq. (3).

In the case of abelian $B F$ theory, this leads to

$$
\begin{aligned}
Z_{\mathrm{Sch}}\left[T, T_{i}\right]= & \operatorname{det}^{\mathrm{b}}\left(d_{1}^{*} d_{1}\right)^{-\frac{1}{4}} \operatorname{det}^{\mathrm{b}}\left(d_{N-2}^{*} d_{N-2}\right)^{-\frac{1}{4}} \operatorname{det}^{\mathrm{b}}\left(d_{0}^{*} d_{0}\right)^{\frac{1}{2}} \\
& \times \operatorname{det}^{\mathrm{b}}\left(d_{N-3}^{*} d_{N-3}\right)^{\frac{1}{2}} \prod_{k=2}^{N-2} \operatorname{det}^{\mathrm{b}}\left(d_{N-(k+2)} d_{N-(k+2)}^{*}\right)^{\frac{(-1)^{k+1}}{2}} .
\end{aligned}
$$


It is possible rewrite the expression for the partition function in a familiar form using the following lemma.

Lemma 29. Let $(M, E, \nabla, \rho)$ be as in Sect. 2.1, then the following holds:

(1) $\operatorname{det}^{\mathrm{b}}\left(d_{k}^{*} d_{k}\right)=\operatorname{det}^{\mathrm{b}}\left(d_{k} d_{k}^{*}\right)$

(2) $\operatorname{det}^{\mathrm{b}}\left(d_{k-1} d_{k-1}^{*}\right)=\operatorname{det}^{\mathrm{b}}\left(d_{N-k}^{*} d_{N-k}\right)$

(3) $\operatorname{det}^{\mathrm{b}}\left(\Delta_{k}\right)=\operatorname{det}^{\mathrm{b}}\left(d_{k-1} d_{k-1}^{*}\right) \operatorname{det}^{\mathrm{b}}\left(d_{k}^{*} d_{k}\right)=\operatorname{det}^{\mathrm{b}}\left(d_{k-1}^{*} d_{k-1}\right) \operatorname{det}^{\mathrm{b}}\left(d_{k}^{*} d_{k}\right)$.

Proof (Sketch of proof). The proof is immediate from the analysis of the spectra of the operators under the consideration. More precisely, note that in this case $\operatorname{det}^{b} \mathrm{~s}$ are spectral invariants as they are the usual zeta-regularised determinants.

(1) follows from the fact that the operators $d_{k}^{*} d_{k}$ and $d_{k} d_{k}^{*}$ are isospectral, a property one can check by acting with $d$ on a coexact eigenform of $\Delta$.

Similarly, we observe that $\left(*_{N-k}\right)^{-1} \circ d_{k-1} d_{k-1}^{*} \circ *_{N-k}=d_{N-k}^{*} d_{N-k}$, which implies that $d_{k-1} d_{k-1}^{*}$ and $d_{N-k}^{*} d_{N-k}$ are isospectral and (2) follows as well.

Finally (3) follows from the observation that the spectrum of $\Delta_{k}=d_{k}^{*} d_{k}+$ $d_{k-1} d_{k-1}^{*}$ is the union of spectrum of $d_{k-1} d_{k-1}^{*}$ and $d_{k}^{*} d_{k}$.

Thanks to the previous lemma, we get:

Proposition 30. The partition function $Z_{S c h}\left[T, T_{i}\right]$ for the resolution (60) yields

$$
Z_{S c h}\left[T, T_{i}\right]=\tau_{\rho}(M) \text {. }
$$

Proof. From (1) and (2) of Lemma 29, we have

$$
\operatorname{det}^{\mathrm{b}}\left(d_{N-(k+2)} d_{N-(k+2)}^{*}\right)=\operatorname{det}^{\mathrm{b}}\left(d_{k+1}^{*} d_{k+1}\right) .
$$

Using this relation and Lemma 29 again, we can rewrite (65) as

$$
\begin{aligned}
& Z_{\text {Sch }}\left[T, T_{i}\right]=\operatorname{det}^{b}\left(d_{1}^{*} d_{1}\right)^{-\frac{1}{4}} \operatorname{det}^{b}\left(d_{N-2}^{*} d_{N-2}\right)^{-\frac{1}{4}} \operatorname{det}^{b}\left(d_{0}^{*} d_{0}\right)^{\frac{1}{2}} \\
& \operatorname{det}^{b}\left(d_{N-3}^{*} d_{N-3}\right)^{\frac{1}{2}} \prod_{k=2}^{N-2} \operatorname{det}^{b}\left(d_{k+1}^{*} d_{k+1}\right)^{\frac{(-1)^{k+1}}{2}} .
\end{aligned}
$$

Now, we know again by Lemma 29 that $\operatorname{det}^{b}\left(d_{1}^{*} d_{1}\right)=\operatorname{det}^{b}\left(d_{N-2}^{*} d_{N-2}\right)$ and $\operatorname{det}^{b}\left(d_{N-3}^{*} d_{N-3}\right)=\operatorname{det}^{b}\left(d_{2}^{*} d_{2}\right)$.

Finally, using (3) of the Lemma 29, we can write

$$
\prod_{k=0}^{N-1} \operatorname{det}^{b}\left(d_{k}^{*} d_{k}\right)^{(-1)^{k} \frac{1}{2}}=\prod_{k=1}^{N} \operatorname{det}^{b}\left(\Delta_{k}\right)^{(-1)^{k+1} \frac{k}{2}}
$$

In summary, we have

$$
Z_{\mathrm{Sch}}\left[T, T_{i}\right]=\prod_{k=1}^{N} \operatorname{det}^{\mathrm{b}}\left(\Delta_{k}\right)^{(-1)^{k+1} \frac{k}{2}},
$$

showing that the partition function $Z_{\mathrm{Sch}}\left[T, T_{i}\right]$ for the resolution (60) of the operator $d$ coincides with the analytic torsion $\tau_{\rho}(M)$ of Definition 13 . 


\subsection{BV Interpretation: Metric Gauge for $B \boldsymbol{F}$ Theory}

We would like to phrase the procedure outlined in Sect. 4.1 in the BatalinVilkovisky framework.

First of all, we need to observe that, when the complex is acyclic, the submanifold

$$
\mathbb{L}_{g}:\left\{d_{\nabla}^{\star} \mathbb{B}=d_{\nabla}^{\star} \mathbb{A}=0\right\}
$$

is Lagrangian in the space of BV fields $\mathcal{F}_{B F}$ (see Remark 5), and can be therefore used as a gauge fixing for $B F$ theory. This is a consequence of Hodge decomposition (on closed manifolds without boundary). In fact, one can easily check that the images of $d$ and $d^{*}$ are both isotropic in $\mathcal{F}_{B F}$, and complementary due to Hodge decomposition. We refer to this gauge fixing as the metric gauge.

Due to the vanishing of the cohomology of $\Omega^{\bullet}(M, E)$, the metric gauge Lagrangian submanifold $\mathbb{L}_{g}$ can be parametrised as follows. The condition $d_{\nabla}^{*} \mathbb{B}=0$ can be equivalently written as $\mathbb{B}=d_{\nabla}^{*} \eta$, and if we specify the form degree of the various fields with a subscript, we write $\mathbb{B}_{N-k-1}=d_{\nabla}^{*} \eta_{N-k}$. Finally, writing $\eta_{N-k}=\star \tau_{k}$, together with the inner product given in Eq. 17 provides the following formula:

$$
\begin{aligned}
\left.\mathbb{S}_{B F}\right|_{\mathbb{L}_{g}} & \equiv \int_{M}\left[\mathbb{B} d_{\nabla} \mathbb{A}_{\mathbb{L}_{g}}^{\text {top }}\right. \\
& =\left.\sum_{k=0}^{N-1} \int_{M} \star d_{\nabla} \tau_{k} d_{\nabla} \mathbb{A}_{k}\right|_{d^{*} \mathbb{A}=0}=\sum_{k=1}^{N}\left(\tau_{k},\left.d_{\nabla}^{*} d_{\nabla}\right|_{\text {coexact }} \mathbb{A}_{k}\right) .
\end{aligned}
$$

Remark 31. We consider an interpretation of Eq. 66 in terms of the BV construction we just outlined. ${ }^{12}$ We will see how the analytic torsion can be seen as a way of making sense of the (super)determinant of the (graded) operator $d: \Omega_{\text {coexact }}^{\bullet} \rightarrow \Omega_{\text {exact }}^{\bullet+1}$. Let us define first

$$
\operatorname{sdet}^{b}\left(d_{\nabla}^{*}\right) \equiv \operatorname{sdet}^{b}\left(d_{\nabla}\right):=\operatorname{sdet}^{b}\left(d_{\nabla}^{*} d_{\nabla}\right)^{\frac{1}{2}}
$$

Definition 9 interprets partition functions of quadratic functionals in terms of the superdeterminant of the operator that features in the functional. However, when an explicit gauge fixing is considered, one needs to take into account the parametrisation of the gauge-fixing Lagrangian. In our case this introduces a "Jacobian superdeterminant" for the change of coordinates $\mathbb{B}=d_{\nabla}^{*} \eta$. Moreover, one should pay attention to the shift in degree $\Omega^{-\bullet}(M, E)[1]$ and $\Omega^{-\bullet}(M, E)[N-2]$. As a matter of fact, in this circumstance the change of variables operator $d^{*}$ is acting on $k$-forms of parity $k+1 \bmod 2$ (instead of $k \bmod 2$ ), so that the Jacobian superdeterminant will appear with the "opposite" power. For the same reason, recalling Remark 12, the output of a Gaussian integration over a 1-shifted graded vector space will yield the superdeterminant of the relevant operator (instead of its inverse).

\footnotetext{
${ }^{12}$ The authors would like to thank P. Mnev and A.S. Cattaneo for valuable insight into this interpretation.
} 
Hence, one recovers the analytic torsion formally as a Gaussian integral on an supervector space by defining the partition function for gauge-fixed $B F$ theory to be a flat-regularised Berezinian/superdeterminant, times the Jacobian (super)determinant $\operatorname{sdet}^{b}\left(d^{*}\right)^{-1}$ of the change of variables operator:

$$
\begin{aligned}
Z\left(\mathcal{S}_{B F}, \mathbb{L}_{g}\right) & =\left.\int_{\mathbb{L}_{g}} \mathrm{e}^{i \mathbb{S}_{B F}}\left|\mathbb{L}_{g}=\right| \operatorname{sdet}^{b}\left(d_{\nabla}^{*}\right)\right|^{-1} \int \exp \left(i \sum_{k=0}^{N-1}\left(\tau_{k},\left(d_{k}^{*} d_{k}\right) \mathbb{A}_{k}\right)\right) \\
& :=\left|\operatorname{sdet}^{b}\left(d_{\nabla}\right)\right|^{-1}\left|\operatorname{sdet}^{b}\left(d_{\nabla}^{*} d_{\nabla}\right)\right|=\left|\operatorname{sdet}^{b}\left(d_{\nabla}^{*} d_{\nabla}\right)\right|^{\frac{1}{2}} \\
& =\prod_{k=0}^{N-1} \operatorname{det}^{b}\left(d_{k}^{*} d_{k}\right)^{(-1)^{k} \frac{1}{2}}=\tau_{\rho}(M)
\end{aligned}
$$

which, comparing with (70), means (cf. [26, Lemma 5.5])

$$
\operatorname{sdet}^{b}\left(d_{\nabla}\right) \equiv \operatorname{sdet}^{b}\left(d_{\nabla}^{*}\right):=\tau_{\rho}(M) .
$$

Observe that, as mentioned in Remark 10, our definition of partition function discards a potential phase factor in Eq. (70), which depends on the signature of the operator. However, as observed in [26, Proposition 5.7 and Remarks $5.8,5.9]$, in the case of acyclic complexes the phase drops out. The analysis of the partition function of $B F$ theory in the metric gauge fixing is discussed in detail in [26], where $B F$ theory is cast on triangulated manifolds with boundary (cellular decompositions of cobordisms) and its partition function is shown to coincide with the Reidermeister torsion. The triangulation of a manifold can also be seen as a way of regularising the infinite-dimensional BV Laplacian $\Delta_{B V}$, of which $\mathbb{S}_{B F}$ is a cocycle.

Remark 32. In Schwarz's formalism, the computation of the partition function is done by performing integration over $\operatorname{ker}(\mathbb{T}) \subset \bigoplus_{j=0}^{N-2} V_{j}$ (see Remark 28). It is tantamount to the 0 -th Koszul-Tate cohomology, i.e. the resolution presented in Eq. (3). This can be compared with the metric gauge in the BV interpretation, where $\mathcal{F}_{B F} \simeq T^{*}[-1] \bigoplus_{k=0}^{N-2} V_{k}[k]$. In order to represent integration over $\operatorname{ker}(\mathbb{T})$ within the $\mathrm{BV}$ formalism, one considers $\mathbb{L}_{g}$, which is essentially the same condition-i.e. restriction to coclosed forms - extended to the shifted cotangent bundle.

\subsection{Contact Gauge Fixing for $B F$ Theory}

This subsection shows that if $B F$ theory is cast on a manifold that admits a contact structure, it is possible to find an alternative gauge-fixing condition. Adopting the same point of view on the partition function, we show how one recovers the Ruelle zeta function.

Consider the geometric data ${ }^{13}$ from Sect. 2.3, $(M, E, X)$, and consider $B F$ theory cast in the Batalin-Vilkovisky formalism as in Definition 27.

\footnotetext{
${ }^{13}$ Again, the importance of $E$ is to ensure acyclicity.
} 
Proposition 33. The submanifold

$$
\mathbb{L}_{X}:=\left\{(\mathbb{A}, \mathbb{B}) \in \mathcal{F}_{B F} \mid \iota_{X} \mathbb{B}=0 ; \iota_{X} \mathbb{A}=0\right\}
$$

is Lagrangian in $\mathcal{F}_{B F}$.

Proof. We show that the condition $\mathbb{L}_{X}: \iota_{X} \mathbb{B}=\iota_{X} \mathbb{A}=0$ defines an isotropic submanifold with an isotropic complement. Indeed, in virtue of the splitting in Eq. (27), we can decompose $\Omega^{\bullet}(M)=\operatorname{ker}\left(\iota_{X}\right) \oplus \operatorname{ker}(\alpha \wedge)$, so that $\mathbb{A}=\varphi+\alpha \wedge \eta$ and $\mathbb{B}=\psi+\alpha \wedge \xi$. We observe that $\eta=\xi=0$ on $\mathbb{L}_{X}$, and defining $\mathbb{L}_{X} \frac{1}{X}:=$ $\left\{(\mathbb{A}, \mathbb{B}) \in \mathcal{F}_{B F} \mid \alpha \wedge \mathbb{A}=\alpha \wedge \mathbb{B}=0\right\}$, also that $\psi=\phi=0$ on $\mathbb{L}_{X} \frac{1}{X}$. Then we have (we understand the top-form part of all the integrands)

$$
\begin{aligned}
\left.\Omega_{B F}\right|_{\mathbb{L}_{X}} & =\int_{M}[\delta \psi \delta(\alpha \wedge \eta)+\delta(\alpha \wedge \xi) \delta \varphi]_{\mathbb{L}_{X}} \equiv 0 \\
\left.\Omega_{B F}\right|_{\mathbb{L}_{X}^{\perp}} & =\int_{M}[\delta \psi \delta(\alpha \wedge \eta)+\delta(\alpha \wedge \xi) \delta \varphi]_{\mathbb{L}_{X}^{\perp}} \equiv 0 .
\end{aligned}
$$

This shows that both $\mathbb{L}_{X}$ and $\mathbb{L}_{X}^{\perp}$ are isotropic.

Definition 34. We shall refer to the choice of gauge fixing proposed in Proposition 33 for $B F$ theory as the contact gauge.

Theorem 35. Consider the geometric data $(M, E, X)$. Suppose that the Ruelle zeta function for $X$ has a meromorphic extension which does not vanish at zero. Then, the Ruelle zeta function at zero $\zeta_{\rho}(0)$ computes the partition function of BF theory in the contact gauge:

$$
Z\left(\mathcal{S}_{B F}, \mathbb{L}_{X}\right)=\left|\zeta_{\rho}(0)\right|^{(-1)^{n+1}} .
$$

Proof. We want to compute $Z\left(\mathcal{S}_{B F}, \mathbb{L}_{X}\right) \equiv Z\left(\left.\mathbb{S}_{B F}\right|_{\mathbb{L}_{X}}\right)$. Because of the decomposition (27), we have that $\iota_{X} \mathbb{B}=0 \Longleftrightarrow \mathbb{B}=(-1)^{|\tau|+1} \iota_{X} \tau$, for some $\tau$; hence,

$$
\begin{aligned}
\left.\mathbb{S}_{B F}\right|_{\mathbb{L}_{X}} & =\int_{M}\left[\mathbb{B} d_{\nabla} \mathbb{A}\right]_{\mathbb{L}_{X}}^{\text {top }}=\int_{M}\left[(-1)^{|\tau|} \iota_{X} \tau d d_{\nabla} \mathbb{A}\right]_{\iota_{X} \mathbb{A}=0}^{\text {top }} \\
& =\int_{M}\left[\tau \iota_{X} d_{\nabla} \mathbb{A}\right]_{\iota_{X} \mathbb{A}=0}^{\text {top }}=\int_{M}\left[\left.\tau \mathcal{L}_{X}\right|_{\Omega_{0}} \mathbb{A}\right]
\end{aligned}
$$

Observe that $\tau$ and $\mathbb{A}$ are inhomogeneous forms in $\alpha \wedge \Omega_{0}^{\bullet}(M, E)[N-2]$ and $\Omega_{0}^{\bullet}(M, E)[1]$, respectively. Then, according to Definition 9 , recalling that $\mathcal{L}_{X}$ acts on a 1-shifted graded vector space and referring to Definition 14 for $\operatorname{sdet}^{b}\left(\iota_{X}\right)$, we have

$$
Z\left(\mathcal{S}_{B F}, \mathbb{L}_{X}\right)=\left|\operatorname{sdet}^{b} \mathcal{L}_{X}\right|_{\Omega_{0}^{\bullet}}|=| \prod_{k=0}^{N-1}\left(\left.\operatorname{det}^{b} \mathcal{L}_{X, k}\right|_{\Omega_{0}^{k}}\right)^{(-1)^{k}} \mid .
$$

This, compared with Eqs. (40) and (50), allows us to conclude the proof. 
Remark 36. We would like to stress how the crucial element in both metric and contact gauges is the existence of a "Hodge decomposition" for the space of $k$-forms. In the metric case, it is given, in particular, by the kernel of the de Rham differential and its dual, but truly it can be considered independently of it, as in the contact case, where the maps $\iota_{X}$ and $\alpha \wedge$ define the splitting.

Remark 3\%. It is tempting to consider a field theory with action functional $S_{X}:=\int_{M}\left[\phi \mathcal{L}_{X} \psi\right]^{\text {top }}$ on differential forms $\phi, \psi \in \Omega^{\bullet}(M)$, and call it Ruelle theory. Observe that this is what one gets out of Theorem 35, but with a nontrivial shift in degree. This is akin to considering the theory $\int_{M}[\mathcal{B} d \mathcal{A}]^{\text {top }}$, for unshifted inhomogeneous differential forms $\mathcal{A}, \mathcal{B} \in \Omega^{\bullet}$, but - to the best of our knowledge - that does not seem to have a clear interpretation up to now.

\section{Lagrangian Homotopies, Fried's Conjecture and Gauge-fixing Independence}

In this section, we outline a strategy to interpret the recent results of [32] on Fried's conjecture as invariance of gauge fixing in the BV formalism, and vice versa. We will set up a general geometric framework to discuss perturbation of the Anosov vector field as an argument for the Ruelle zeta function, and argue how this can be used to construct homotopies for Lagrangian submanifolds. Indeed, consider the following:

Claim 38. Assume Theorem 2 holds on $\mathcal{F}_{B F}$ for some appropriate choice of $B V$ Laplacian $\Delta_{B V}$, and assume there exists a Lagrangian homotopy between $\mathbb{L}_{X}$ and $\mathbb{L}_{g}$ for any $X$ contact and Anosov. Then, up to phase,

$$
\tau_{\rho}(M)=Z\left(\mathcal{S}_{B F}, \mathbb{L}_{g}\right)=Z\left(\mathcal{S}_{B F}, \mathbb{L}_{X}\right)=\left|\zeta_{\rho}(0)\right|^{(-1)^{n}},
$$

which is the statement of (Fried's) Conjecture 21.

Remark 39. We stress here that a universal generalisation of Theorem 2 to infinite dimensions, i.e. one that works independently of the details of the field theory (and gauge fixing), is currently not yet available. The results of [26] suggest that one possible regularisation scheme for the BV Laplacian might arise as a limit of finite-dimensional cellular analogues. The works of [27] and $[40,44]$ assume instead a somewhat different set-up, which would also need to be adapted to the case at hand. Ideally, a scheme should be found that makes the regularisation of the BV Laplacian compatible with the context of Definition 7. Observe that the recent results of [20] suggest that a number of subtleties might arise, already in acyclic cases.

The central notions for this section, and the main theorem we will need are as follows.

Definition 40. Let $X$ be an Anosov vector field on a manifold $M$. We will say that $X$ is regular whenever the restricted resolvents $R_{k}(\lambda)=\left(\lambda_{X, k}+\lambda\right)^{-1}$ of Eq. (52) do not have poles at $\lambda=0$ for all $0 \leq k \leq N-1$. 
Recalling Remark 22, we report now a result by Dang, Guillarmou, Rivière and Shen on the properties of Ruelle zeta function seen as a function on Anosov vector fields:

Theorem 41 ([32]). Let $(M, E)$ denote the geometric data of Sect. 2.1. Consider the set $U \subset C^{\infty}(M, T M)$ of regular smooth Anosov vector fields $X$. Then this set is open, and the $\operatorname{map} \zeta: U \rightarrow \mathbb{C}$, sending $X$ to $\zeta(X, 0)$, is locally constant and nonzero.

\subsection{A Sphere Bundle Construction}

Let $\Sigma$ be a compact manifold. We denote by $\mathcal{R}(\Sigma)$ the space of all Riemannian metrics on $\Sigma$, by $\mathcal{R}_{<}(\Sigma)$ the space of negative sectional curvature metrics, and by $\mathcal{R}_{h}(\Sigma)$ the space of hyperbolic metrics.

Definition 42. Let us fix a reference metric $g_{0}$. We denote the sphere bundle associated with $g_{0}$ by $S_{0}^{*} \Sigma:=S_{g_{0}}^{*} \Sigma$. Moreover, let $g, \widetilde{g} \in \mathcal{R}(\Sigma)$ and consider the diffeomorphism

$$
\sigma_{g}^{\widetilde{g}}: S_{g}^{*} \Sigma \longrightarrow S_{\widetilde{g}}^{*} \Sigma
$$

obtained by rescaling lengths in $T \Sigma$. Then, we denote the diffeomorphisms $\sigma_{g}: S_{g}^{*} \Sigma \rightarrow S_{0}^{*} \Sigma$, with $\sigma_{g}:=\sigma_{g}^{g_{0}}$ for all $g \in \mathcal{R}(\Sigma)$. Denoting by $\varphi_{g}$ the geodesic flow on $S_{g}^{*} \Sigma$, and by $X_{g}$ the associated vector field. One can transfer the geodesic flow $\varphi_{g}$ to $S_{0}^{*} \Sigma$ by $\varphi_{g}^{0}=\sigma_{g} \circ \varphi_{g} \circ \sigma_{g}^{-1}$; we denote the associated vector field on $S_{0}^{*} \Sigma$ by $X_{g}^{0}$.

Since we are interested in computing the Ruelle zeta function on sphere bundles, we would like to ensure that the geodesic (Reeb) vector field $X_{g}$ is Anosov in $S_{g}^{*} \Sigma$. This would not be true in general, but it will be true for metrics of negative sectional curvature $\mathcal{R}_{<}$. The map $\sigma_{g}$ allows us to consider all Anosov geodesic vector fields on the same reference space $S_{0}^{*} \Sigma$.

Lemma 43. The Anosov property of is preserved under pushforward by $\sigma_{g}$.

Proof. This is an adaptation of a result proved in [49]. Given $S_{g}^{*} \Sigma$ with Anosov flow $\varphi_{g}$ and associated vector field $X_{g}$, we also have a decomposition

$$
T\left(S_{g}^{*} \Sigma\right)=\mathbb{R} X_{g}+E_{s}\left(X_{g}\right)+E_{u}\left(X_{g}\right)
$$

with constants $C_{g}, \lambda_{g}$ so that for all $v_{s} \in E_{s}\left(X_{g}\right), v_{u} \in E_{u}\left(X_{g}\right)$ and all $t>0$ :

$$
\left\|\mathrm{d} \varphi_{g, t} v_{s}\right\|_{g} \leq C_{g} \mathrm{e}^{-\lambda_{g} t}\left\|v_{s}\right\|_{g}, \quad\left\|\mathrm{~d} \varphi_{g,-t} v_{u}\right\|_{g} \leq C_{g} \mathrm{e}^{-\lambda_{g} t}\left\|v_{u}\right\|_{g}
$$

Using $\mathrm{d} \sigma_{g}$, we push the decomposition of $T\left(S_{g}^{*} \Sigma\right)$ to $T\left(S_{0}^{*} \Sigma\right)$ providing

$$
T\left(S_{0}^{*} \Sigma\right)=\mathbb{R} X_{g}^{0}+E_{s}\left(X_{g}^{0}\right)+E_{u}\left(X_{g}^{0}\right)
$$

where $E_{s}\left(X_{g}^{0}\right):=\mathrm{d} \sigma_{g}\left(E_{s}\left(X_{g}\right)\right)$ and $E_{u}\left(X_{g}^{0}\right):=\mathrm{d} \sigma_{g}\left(E_{u}\left(X_{g}\right)\right)$. Now $\mathrm{d} \sigma_{g}$ and $\mathrm{d} \sigma_{g}^{-1}$ are both bounded so for $v_{s}^{0} \in E_{s}\left(X_{g}^{0}\right)$, there is $v_{s} \in E_{s}$ such that $\mathrm{d} \sigma_{g} v_{s}=$ 
$v_{s}^{0}$, whence for $t>0$ :

$$
\begin{aligned}
& \left\|\mathrm{d} \varphi_{g, t}^{0} v_{s}^{0}\right\|_{0} \\
& \quad=\left\|\mathrm{d} \sigma_{g} \mathrm{~d} \varphi_{g, t} v_{s}\right\|_{0} \leq\left\|\mathrm{d} \sigma_{g}\right\|\left\|\mathrm{d} \varphi_{g, t} v_{s}\right\|_{g} \leq\left\|\mathrm{d} \sigma_{g}\right\| C_{g} \mathrm{e}^{-\lambda_{g} t}\left\|v_{s}\right\|_{g} \\
& \quad=\left\|\mathrm{d} \sigma_{g}\right\| C_{g} \mathrm{e}^{-\lambda_{g} t}\left\|\mathrm{~d} \sigma_{g}^{-1} v_{s}^{0}\right\|_{g} \leq\left\|\mathrm{d} \sigma_{g}\right\|\left\|\mathrm{d} \sigma_{g}^{-1}\right\| C_{g} \mathrm{e}^{-\lambda_{g} t}\left\|v_{s}^{0}\right\|_{0}=C_{g}^{\prime} \mathrm{e}^{-\lambda_{g} t}\left\|v_{s}^{0}\right\|_{0} .
\end{aligned}
$$

A similar result holds for vectors in $E_{u}\left(X_{g}^{0}\right)$.

Definition 44. We define the assignment:

$$
\mathbb{X}: \mathcal{R}(\Sigma) \longrightarrow C^{\infty}\left(S_{0}^{*} \Sigma ; T\left(S_{0}^{*} \Sigma\right)\right) ; \quad \mathbb{X}(g)=\mathrm{d} \sigma_{g} X_{g}=X_{g}^{0}
$$

where $X_{g}$ is the geodesic vector field induced by $g$ on $S_{g}^{*} \Sigma$.

Proposition 45. Let us denote by $\mathcal{A}(\Sigma)$ the set of Anosov vector fields on $S_{0}^{*} \Sigma$, and by $\mathcal{R}_{<}(\Sigma)$ the space of negative sectional curvature metrics on $\Sigma$. Then

$$
\mathcal{A}_{<}(\Sigma):=\operatorname{Im}\left(\left.\mathbb{X}\right|_{\mathcal{R}_{<}(\Sigma)}\right) \subset \mathcal{A}(\Sigma) .
$$

Proof. This follows from the general fact that all negative sectional curvature metrics have Anosov geodesic flows, and application of Lemma 43.

Lemma 46. For $\Sigma$ a two-dimensional surface of negative Euler characteristic $\chi(\Sigma)<0$, the image of $\mathbb{X}$ restricted to $\mathcal{R}_{h}(\Sigma)$, the space of hyperbolic metrics on $\Sigma$, is a deformation retract of $\mathcal{A}_{<}(\Sigma)$.

Proof. On two-dimensional surfaces, the space of negative sectional curvature metrics coincides with the space of negative Ricci curvature metrics, which is a contractible subset of $\mathcal{R}$. In fact, via the Ricci flow one canonically deforms any metric $g$ into a hyperbolic one, and in particular, negatively curved metrics are deformed in such a way along a path of negatively curved metrics [60]. Thus, the space of hyperbolic metrics on $\Sigma$ is a deformation retract of the space of all negatively curved metrics $\mathcal{R}_{<}(\Sigma)$, and so will be its image under $\mathbb{X}$ with respect to the space $\mathcal{A}_{<}(\Sigma)$.

Theorem 47. Consider the contact Anosov-Riemannian structure $\left(M, E, X_{g}, g\right)$ of Sect. 2.4.1, where $M=S_{g}^{*} \Sigma$ with $\operatorname{dim}(\Sigma)=2$, the Euler characteristic $\chi(\Sigma)<0$, and $g \in \mathcal{R}_{<}(\Sigma)$. Then,

$$
\left|\zeta\left(X_{g}, 0\right)\right|=\tau_{\rho}(M)^{-1} .
$$

Proof. In virtue of Lemma 46, there exists a smooth path $X(t)$ of Anosov vector fields in $\mathcal{A}_{<}(\Sigma)$ connecting $X_{g}$ to $X_{g_{h}}$ for some $g_{h}$ hyperbolic, where Theorem 20 holds: let $g(t)$ be a retraction such that $g(0)=g_{0}$ and $g(1)=g$, and set $X(t)=\mathbb{X}(g(t))$. If we can show that each $X(t)$ is regular, then local constancy of the zeta function at zero (Theorem 41) provides the result.

The idea for showing regularity is essentially in [34] even though that setting is using the trivial representation, so that the de Rham complex is not acyclic. A more explicit version which adapts the spirit of [34] is in [32]. Below we cite the necessary results of [32] to conclude that $X(t)$ is regular. 
Since $g \in \mathcal{R}_{<}(\Sigma)$, its associated geodesic vector field $X_{g}$ on $S_{g}^{*} \Sigma$ is Anosov $[1,5,6]$. For regularity, one shows that no $k$-form resonant states $\varphi^{(k)}$ associated with the spectral parameter $\lambda=0$ exist for $k \in\{0,1,2\}$ (recall Eq. (54)). By [32, Lemma 7.4], 0-form resonant states $\varphi^{(0)}$ are closed and smooth in this setting so may be identified with degree-0 de Rham cohomology. Acyclicity implies such states are necessarily the 0 -section. For 2-form resonant states $\varphi^{(k)}$, there is an isomorphism with 0 -form resonant states upon wedging with $\mathrm{d} \alpha$ [32, Lemma 7.2]. Effectively $\varphi^{(2)}=\varphi^{(0)} \mathrm{d} \alpha$ so in this case also, no such resonant states exist. Finally, for 1 -form resonant states, we require degree1 cohomology to vanish and appeal to [32, Lemma 7.2 Hypothesis (1)] to conclude no nontrivial resonant states exist.

Corollary 48. Under the assumptions of Theorem 47, denoting by

$$
\mathbb{L}_{X}=\left\{(\mathbb{A}, \mathbb{B}) \in \mathcal{F}_{B F} \mid \iota_{\mathbb{X}(g)} \mathbb{A}=\iota_{\mathbb{X}(g)} \mathbb{B}=0\right\}
$$

the Lagrangian submanifold defined by the Anosov vector field $\mathbb{X}(g)$, we have

$$
Z\left(\mathcal{S}_{B F}, \mathbb{L}_{X}\right)=\tau_{\rho}(M)^{-1},
$$

for every $g \in \mathcal{R}_{<}(\Sigma)$.

Proof. This follows from Theorems 35 and 47.

Remark 49. Observe that the above construction can be generalised to some extent. In general, the space of negative sectional curvature metric will not be path connected, so let us consider the connected components that contain a hyperbolic metric. The image under $\mathbb{X}$ of the disjoint union of such connected components in $\mathcal{A}(\Sigma)$ will be a union of islands of Anosov vector fields, path connected to an Anosov vector fields in $\operatorname{Im}\left(\left.\mathbb{X}\right|_{\mathcal{R}_{h}}\right)$. In a neighbourhood of a hyperbolic metric Anosov vector field, the requirements of Theorem 41 are satisfied, and Fried's conjecture might be extended to open subsets of $\mathcal{A}_{<}(\Sigma)$. We defer the development of such a generalisation to a subsequent work.

Remark 50. The spirit of gauge-fixing homotopies is that of replacing an illdefined integral with a well-defined one, and can be considered as providing a family of integral representations of the same quantity, only some of which are directly computable. From this point of view, the Ruelle zeta function at zero might not be computable for a generic (Anosov) vector field, but it will be once deformed away from an invalid point in $\mathcal{A}(\Sigma)$.

We wish to interpret this result in terms of homotopies of Lagrangian submanifolds in $\mathcal{F}_{B F}$ and gauge-fixing independence for $B F$ theory.

Theorem 51. Consider the geometric data $\left(M, E, X_{g}, g\right)$ of Sect. 2.4.1, a smooth path $g_{t}:[0,1] \rightarrow \mathcal{R}(\Sigma)$ such that $\mathbb{X}\left(g_{0}\right)$ is regular, and let

$$
\mathbb{L}_{t}:=\left\{(\mathbb{A}, \mathbb{B}) \in \mathcal{F}_{B F} \mid \iota_{\mathbb{X}\left(g_{t}\right)} \mathbb{A}=\iota_{\mathbb{X}\left(g_{t}\right)} \mathbb{B}=0\right\}
$$

be the associated smooth family of Lagrangian submanifolds in $\mathcal{F}_{B F}$. Then, $Z\left(\mathcal{S}_{B F}, \mathbb{L}_{0}\right) \neq 0$ and

$$
\left.\frac{d}{\mathrm{~d} t}\right|_{t=0} Z\left(\mathcal{S}_{B F}, \mathbb{L}_{t}\right)=0 .
$$


Moreover, if $g_{0}$ is hyperbolic, we have that

$$
Z\left(\mathcal{S}_{B F}, \mathbb{L}_{0}\right)=\tau_{\rho}(M) .
$$

Proof. Lemma 43 ensures that $\mathbb{X}\left(g_{t}\right)=\mathrm{d} \sigma_{g_{t}} X_{g_{t}}$ is a smooth path of Anosov vector fields in $S_{0}^{*} \Sigma$, and in virtue of Theorem 35 , we have that

$$
Z\left(\mathcal{S}_{B F}, \mathbb{L}_{t}\right) \equiv Z\left(\left.\mathbb{S}_{R}\right|_{\mathbb{L}_{t}}\right)=\left|\zeta\left(\mathbb{X}\left(g_{t}\right), 0\right)\right|^{(-1)^{n}} .
$$

By assumption, at $g_{0}$ the $k$ th Ruelle zeta factors $\zeta_{k}\left(\mathbb{X}\left(g_{0}\right), 0\right)$ are well defined and different from zero. Then, in virtue of Theorem $41 \zeta\left(\mathbb{X}\left(g_{0}\right), 0\right)$ is constant in a open neighbourhood of $\mathbb{X}\left(g_{0}\right)$; hence, it is on the whole path $g_{t}$ for $t \in[0, T)$ for some appropriately chosen $T$, and in particular, its derivative at $t$ vanishes. If we choose $g_{0}$ hyperbolic, using Theorem 20 we can conclude that

$$
Z\left(\mathcal{S}_{B F}, \mathbb{L}_{0}\right)=\left|\zeta\left(\mathbb{X}\left(g_{0}\right), 0\right)\right|^{(-1)^{n}} \equiv\left|\zeta_{\rho}(M)\right|^{(-1)^{n}}=\tau_{\rho}(M) .
$$

\section{Acknowledgements}

This research was (partly) supported by the NCCR SwissMAP, funded by the Swiss National Science Foundation. MS acknowledges partial support from Swiss National Science Foundation Grants P2ZHP2_164999 and P300P2_ 177862. SK acknowledges partial support from Deutsche Forschungs Gemeinschaft (DFG) under Graduiertenkolleg 1821 (Cohomological Methods in Geometry). This project began, and was mostly developed, during the period when the first and last author were at UC Berkeley, to which we are grateful for having provided such a stimulating research environment. M.S. would like to thank the University of Freiburg for hospitality. We are indebted to A.S. Cattaneo and P. Mnev for their support and feedback at different stages of this work. Since the preprint appeared on arXiv, we have received several useful comments from Y. Chaubet and N.V. Dang whom we warmly thank. We also thank the anonymous referee for providing useful comments that helped improve this paper.

Funding Open access funding provided by Swiss Federal Institute of Technology Zurich.

Open Access. This article is licensed under a Creative Commons Attribution 4.0 International License, which permits use, sharing, adaptation, distribution and reproduction in any medium or format, as long as you give appropriate credit to the original author(s) and the source, provide a link to the Creative Commons licence, and indicate if changes were made. The images or other third party material in this article are included in the article's Creative Commons licence, unless indicated otherwise in a credit line to the material. If material is not included in the article's Creative Commons licence and your intended use is not permitted by statutory regulation or exceeds the permitted use, you will need to obtain permission directly from 
the copyright holder. To view a copy of this licence, visit http://creativecommons. org/licenses/by/4.0/.

Publisher's Note Springer Nature remains neutral with regard to jurisdictional claims in published maps and institutional affiliations.

\section{References}

[1] Arnold, V.I., Avez, A.: Problèmes ergodiques de la mécanique classique, Paris, Gauthier-Villars (1967); (English translation: Ergodic problems in classical mechanics) New York (1968)

[2] Atiyah, M.F., Bott, R.: Notes on the Lefschetz Fixed Point Theorem for Elliptic Complexes. Harvard University, Cambridge (1964)

[3] Atiyah, M.F., Bott, R.: A Lefschetz fixed point formula for elliptic complexes: II. Applications. Ann. Math. 88, 451-491 (1968)

[4] Anderson, I.M.: Introduction to the variational bicomplex. Contemp. Math. 132, $51(1992)$

[5] Anosov, D.V.: Geodesic flows on closed Riemannian manifolds of negative curvature. Trudy Mat. Inst. Steklov 90(5), 3-210 (1967)

[6] Anosov, D.V., Sinai, Y.G.: Some smooth ergodic systems. Russ. Math. Surv. 22(5), 103 (1967)

[7] Baladi, V.: Dynamical Zeta Functions and Dynamical Determinants for Hyperbolic Maps. Springer, Berlin (2018)

[8] Birmingham, D., Blau, M., Rakowski, M., Thompson, G.: Topological field theory. Phys. Rep. 209(4), 129-340 (1991)

[9] Berwick-Evans, D.: The Chern-Gauss-Bonnet theorem via supersymmetric Euclidean field theories. Commun. Math. Phys. 335(3), 1121-1157 (2015)

[10] Berezin, F.A.: Introduction to Algebra and Analysis with Anticommuting Variables. Moscow University, Moscow (1983)

[11] Berezin, F.A., Leites, D.A.: Supermanifolds. Doklady Akademii Nauk 224, 505508 (1975)

[12] Butterley, O., Liverani, C.: Smooth Anosov flows: correlation spectra and stability. J. Mod. Dyn. 1(2), 301-322 (2007)

[13] Becchi, C., Rouet, A., Stora, R.: The abelian Higgs Kibble model, unitarity of the s-operator. Phys. Lett. B 52(3), 344-346 (1974)

[14] Becchi, C., Rouet, A., Stora, R.: Renormalization of the abelian Higgs-Kibble model. Commun. Math. Phys. 42(2), 127-162 (1975)

[15] Becchi, C., Rouet, A., Stora, R.: Renormalization of gauge theories. Ann. Phys. 98(2), 287-321 (1976)

[16] Batalin, I.A., Vilkovisky, G.A.: Quantization of gauge theories with linearly dependent generators. Phys. Rev. D 28(10), 2567 (1983)

[17] Batalin, I.A., Vilkovisky, G.A.: Gauge algebra and quantization. Quantum Gravity, pp. 463-480. Springer, Berlin (1984)

[18] Cattaneo, A.S., Contreras, I.: Split canonical relations (2018). arXiv:1811.10107

[19] Cattaneo, A.S., Cotta-Ramusino, P., Fröhlich, J., Martellini, M.: Topological BF theories in 3 and 4 dimensions. J. Math. Phys. 36(11), 6137-6160 (1995) 
[20] Chaubet, Y., Dang, N.V.: Dynamical torsion for contact Anosov flows (2019). arXiv:1911.09931

[21] Cattaneo, A.S., Fiorenza, D., Longoni, R.: Graded Poisson algebras. In: Françoise, J.P., Naber, G.L., Tsun, T.S. (eds.) Encyclopedia of Mathematical Physics, pp. 560-567. Academic Press, Oxford (2006)

[22] Costello, K., Gwilliam, O.: Factorization Algebras in Quantum Field Theory, vol. 1. Cambridge University Press, Cambridge (2016)

[23] Cordes, S., Moore, G., Ramgoolam, S.: Lectures on 2d Yang-Mills theory, equivariant cohomology and topological field theories. Nucl. Phys. B Proc. Suppl. 41(1), 184-244 (1995)

[24] Cattaneo, A.S., Mnev, P., Reshetikhin, N.: Classical BV theories on manifolds with boundary. Commun. Math. Phys. 332(2), 535-603 (2014)

[25] Cattaneo, A.S., Mnev, P., Reshetikhin, N.: Perturbative quantum gauge theories on manifolds with boundary. Commun. Math. Phys. 357(2), 631-730 (2018)

[26] Cattaneo, A.S., Mnev, P., Reshetikhin, N.: A cellular topological field theory. Commun. Math. Phys. 374(2), 1229-1320 (2020)

[27] Costello, K.: Renormalization and Effective Field Theory, vol. 170. American Mathematical Society, Providence (2011)

[28] Cattaneo, A.S., Rossi, C.A.: Higher-dimensional BF theories in the BatalinVilkovisky formalism: the BV action and generalized Wilson loops. Commun. Math. Phys. 221(3), 591-657 (2001)

[29] Cattaneo, A.S., Schätz, F.: Introduction to supergeometry. Rev. Math. Phys. 23(06), 669-690 (2011)

[30] Delgado, N.L.: Lagrangian field theories: ind/pro-approach and L-infinity algebra of local observables. PhD thesis, Max Planck Institute for Mathematics (2017)

[31] Deligne, P., Freed, D.S.: Classical field theory. Quantum Fields and Strings: A Course for Mathematicians, vol. 2, pp. 137-226. American Mathematical Society, Providence (1999)

[32] Dang, N.V., Guillarmou, C., Rivière, G., Shen, S.: The Fried conjecture in small dimensions. Invent. Math. 220(2), 525-579 (2020)

[33] Dyatlov, S., Zworski, M.: Dynamical zeta functions for Anosov flows via microlocal analysis. Ann. Sci. Éc. Norm. Supér. (4) 49(3), 543-577 (2016)

[34] Dyatlov, S., Zworski, M.: Ruelle zeta function at zero for surfaces. Invent. Math. 210(1), 211-229 (2017)

[35] Faddeev, L.D., Popov, V.N.: Feynman diagrams for the Yang-Mills field. Fifty Years of Mathematical Physics: Selected Works of Ludwig Faddeev, pp. 157-158. World Scientific, Singapore (2016)

[36] Fredenhagen, K., Rejzner, K.: Batalin-Vilkovisky formalism in perturbative algebraic quantum field theory. Commun. Math. Phys. 317(3), 697-725 (2013)

[37] Fried, D.: Analytic torsion and closed geodesics on hyperbolic manifolds. Invent. Math. 84(3), 523-540 (1986)

[38] Fried, D.: Lefschetz formulas for flows. Contemp. Math. 58, 19-69 (1987)

[39] Fried, D.: Meromorphic zeta functions for analytic flows. Commun. Math. Phys. 174(1), 161-190 (1995) 
[40] Gwilliam, O., Haugseng, R.: Linear Batalin-Vilkovisky quantization as a functor of $\infty$-categories. Sel. Math. New Ser. 24(2), 1247-1313 (2018)

[41] Giulietti, P., Liverani, C., Pollicott, M.: Anosov flows and dynamical zeta functions. Ann. Math. 178, 687-773 (2013)

[42] Guillemin, V.: Lectures on spectral theory of elliptic operators. Duke Math. J. 44(3), 485-517 (1977)

[43] Gutzwiller, M.C.: Chaos in Classical and Quantum Mechanics, vol. 1. Springer, Berlin (1991)

[44] Gwilliam, O.: Factorization algebras and free field theories. PhD thesis, Northwestern University

[45] Hadfield, C.S.: Zeta function at zero for surfaces with boundary (2018). arXiv:1803.10982

[46] Henneaux, M.: Lectures on the antifield-BRST formalism for gauge theories. Nucl. Phys. B Proc. Suppl. 18(1), 47-105 (1990)

[47] Küster, B., Weich, T.: Pollicott-Ruelle resonant states and Betti numbers. Commun. Math. Phys. 378(2), 917-941 (2020)

[48] Marklof, J.: Selberg's trace formula: an introduction. In: Bolte, J., Steiner, F. (eds.) Hyperbolic Geometry and Applications in Quantum Chaos and Cosmology, pp. 83-119. Cambridge University Press, Cambridge (2011)

[49] Matsumoto, S.: The space of (contact) Anosov flows on 3-manifolds. J. Math. Sci. Univ. Tokyo 20, 445-460 (2013)

[50] Mnev, P.: Lecture notes on torsions (2014). arXiv:1406.3705

[51] Mnev, P.: Quantum Field Theory: Batalin-Vilkovisky Formalism and Its Applications, vol. 72. American Mathematical Society, Providence (2019)

[52] Moscovici, H., Stanton, R.J.: R-torsion and zeta functions for locally symmetric manifolds. Invent. Math. 105(1), 185-216 (1991)

[53] Ray, D.B., Singer, I.M.: R-torsion and the Laplacian on Riemannian manifolds. Adv. Math. 7(2), 145-210 (1971)

[54] Ruelle, D.: Zeta-functions for expanding maps and Anosov flows. Invent. Math. 34(3), 231-242 (1976)

[55] Ruelle, D.: Resonances of chaotic dynamical systems. Phys. Rev. Lett. 56(5), 405 (1986)

[56] Schwarz, A.S.: The partition function of degenerate quadratic functional and Ray-Singer invariants. Lett. Math. Phys. 2(3), 247-252 (1978)

[57] Schwarz, A.S.: The partition function of a degenerate functional. Commun. Math. Phys. 67(1), 1-16 (1979)

[58] Shen, S.: Analytic torsion, dynamical zeta functions, and the Fried conjecture. Anal. PDE 11(1), 1-74 (2017)

[59] Stasheff, J.: The (secret?) homological algebra of the Batalin-Vilkovisky approach. In: Conference Secondary Calculus and Cohomological Physics, August 1998, vol. 219, pp. 195-210. Moscow (1998)

[60] Tuschmann, W., Wraith, D.J.: Moduli Spaces of Riemannian Metrics. Springer, Berlin (2015)

[61] Tyutin, I.V.: Gauge invariance in field theory and statistical physics in operator formalism. Lebedeiv Physics Institute preprint (1975). arXiv:0812.0580 
[62] Voronov, T.: Geometric Integration Theory on Supermanifolds, vol. 1. CRC Press, Boca Raton (1991)

[63] Weinstein, A.: Symplectic manifolds and their Lagrangian submanifolds. Adv. Math. 6(3), 329-346 (1971)

[64] Weinstein, A.: Symplectic categories. Port. Math. 67(2), 261-278 (2010)

[65] Zworski, M.: Mathematical study of scattering resonances. Bull. Math. Sci. 7(1), $1-85$ (2017)

Charles Hadfield

IBM T.J. Watson Research Center

1101 Kitchawan Rd

Yorktown Heights NY 10598

USA

e-mail: charles.hadfield@ibm.com

Santosh Kandel

Mathematics Institute

University of Freiburg

79104 Freiburg

Germany

e-mail: skandel1@alumni.nd.edu

Michele Schiavina

Department of Mathematics

ETH Zürich

Rämistrasse 101

8092 Zurich

Switzerland

and

Institute for Theoretical Physics

ETH Zürich

Wolfgang-Pauli strasse 27

8093 Zurich

Switzerland

e-mail: micschia@phys.ethz.ch

Communicated by Christoph Kopper.

Received: May 15, 2020.

Accepted: September 23, 2020. 